Jan PALCZEWski (Leeds and Warszawa)

ŁukAsz SteTtNeR (Warszawa)

\title{
GROWTH-OPTIMAL PORTFOLIOS UNDER TRANSACTION COSTS
}

Abstract. This paper studies a portfolio optimization problem in a discrete-time Markovian model of a financial market, in which asset price dynamics depends on an external process of economic factors. There are transaction costs with a structure that covers, in particular, the case of fixed plus proportional costs. We prove that there exists a self-financing trading strategy maximizing the average growth rate of the portfolio wealth. We show that this strategy has a Markovian form. Our result is obtained by large deviations estimates on empirical measures of the price process and by a generalization of the vanishing discount method to discontinuous transition operators.

1. Introduction. Researchers and practitioners have long been aware that Markovian models of asset price dynamics, such as the Cox-RossRubinstein model or the Black-Scholes model, have significant deficiencies related to non-stationarity of the financial market. They observed that the volatility and the expected rate of return of asset prices are not constant but depend on the economic situation, which may change over longer time spans. As a remedy, they introduced additional processes modelling vital market variables, such as market trend or price volatility. However, a unified framework has only recently been introduced and has attracted a lot of interest (see e.g. [5], [6], [12], [26], [25], [31]). Existing literature concentrates mainly on continuous-time diffusion models. Bielecki et al. [6] solve an asset management problem where economic factors, as those additional market variables

2000 Mathematics Subject Classification: Primary 91B28; Secondary 93E20.

Key words and phrases: portfolio optimization, transaction costs, growth rate, logarithmic utility, impulsive strategy.

Research supported by MNiSzW grant P03A 01328. 
are called, form a diffusion that is independent of the Brownian motion governing the price process and they affect only the drift of the price process. Fleming and Sheu [12] allow both processes to have dependent Brownian motions but their diffusions are of a special form. Palczewski and Stettner [26], though, assume only that asset prices and economic factors follow one general continuous-time Markov process and prove results concerning optimal portfolio selection for an infinite time-discounted performance functional under transaction costs.

In the present paper we study a portfolio management problem in which performance is measured by an average growth rate of the portfolio wealth. We work within a discrete time framework which allows us to overcome limitations and technicalities of the existing theory of continuous time Markov processes and impulsive control. The market consists of $d$ assets, whose prices are, in general, interdependent. Their dynamics are affected by a process of economic factors, which is a Markov process on a Polish space (for details see Section 2). We assume that assets cannot go bankrupt (their prices are positive). We impose costs of performing transactions. These costs, in the simplest, consist of a fixed part, independent of the transaction, and a proportional part, depending on the volume and type of assets sold or purchased (see (4), (5) and the following discussion). This type of transaction costs prevents continuous trading in continuous-time models (see e.g. [26]) and emulates existing market mechanisms. The framework of this paper covers more general transaction costs structures as well (see Section 6). Performance of a portfolio $\Pi$ is measured by the functional

$$
J(\Pi)=\liminf _{T \rightarrow \infty} \frac{1}{T} \mathbb{E} \ln X^{\Pi}(T),
$$

where $X^{\Pi}(T)$ is the wealth of the portfolio $\Pi$ at time $T$. This functional computes an average growth rate of the portfolio $\Pi$ as can be seen from the following reformulation of the above formula:

$$
J(\Pi)=\liminf _{T \rightarrow \infty} \frac{1}{T} \mathbb{E} \sum_{k=0}^{T-1} \ln \frac{X^{\Pi}(k+1)}{X^{\Pi}(k)} .
$$

The aim of this paper is to find a portfolio that maximizes the value of (1). This is an infinite-time counterpart of the logarithmic utility maximization, which is widely used in the economic and financial community, where optimal portfolios are referred to as log-optimal or growth-optimal. For a broader treatment see textbooks [10], [24]. In mathematical context the research goes back to Kelly (see [21], [32]) and has continued in discrete time ([3]) and continuous time ([1], [2]) till the present day ([13], [17], [27]). Functional (1) can also be seen as a risk sensitive functional and the literature is here broad as well ([6], [22], [31]). It should be stressed that most of the papers consider 
continuous time diffusion models, where an optimal strategy is obtained as a solution to an appropriate HJB equation, usually reformulated in a variational form. Consequently, the results are based on a sophisticated theory of PDEs and solutions usually do not use directly probabilistic properties of the phenomena under study. Moreover, due to complexity of the studied PDEs the results are often of existential form.

In this paper, we approach the optimization problem (1) from a probabilistic point of view. We prove that there exists a self-financing portfolio strategy maximizing the growth rate (1). We show that this trading strategy has a Markovian form, i.e. an investment decision at time $t$ is based only on the state of asset prices and economic factors at $t$. Main additions to the existing theory are transaction costs with a fixed term and a general form of dependence of asset prices on economic factors. As far as we know there is no paper that treats this type of problem in such generality.

Our study depends strongly on the reformulation (2) of the performance functional. It exposes the Markovian structure of the functional and allows application of the theory of optimization of long-run average cost functionals. A survey of standard methods for long-run average cost functionals is in [4]. We will, however, borrow from a new technique invented by Schäl [28], who initiated the use of Bellman inequalities leading to significantly more general results. His ideas thrive in [16] (weighted norms), [14] (stochastic games) and recently in [19]. Those results strongly depend on continuity properties of the controlled transition operator of the Markov process under consideration. In this paper we show that the above ideas can also be used in the study of problems which fail to satisfy the continuity assumptions. Moreover, following [19] we are able to remove a requirement for the state space to be locally compact as is needed in the seminal paper [28]. This significantly generalizes the applicability of this framework to incomplete information case (for details on the incomplete information model see Section 6 and [25]).

The paper is organized as follows. In Section 2 we introduce the model. We specify the dynamics of the asset price process and the form of transaction costs. We introduce a process representing proportions of the portfolio wealth invested in the individual assets and we reformulate the initial problem in terms of the proportions. This reformulation plays a major role in the paper.

In Section 3 the main assumptions are listed. We prove ergodicity results and large deviation estimates on empirical measures of the price process. They give a new insight into the dynamics of the price process.

The study of value functions of discounted functionals related to (2) is pursued in Section 4. A few important technical results on the consequences 
of the transaction costs are stated (their proofs are in Appendix). They are used to build a relation between value functions for the discounted problems with and without the fixed term in the transaction costs structure. This is a starting point for derivation of the Bellman inequality, which is done in Section 5. At this stage we also deal with the lack of continuity of the controlled transition operator. We prove the existence of a growth-optimal strategy and show its form. We also relate the results to the case without constant term in the transaction costs structure (see [31]).

Section 6 presents extensions of our results to other transaction costs structures and shows how existing results can be used in the case of incomplete observation of economic factors.

2. Preliminaries. The market model is constructed on a probability space $(\Omega, \mathcal{F}, \mathbb{P})$. Prices of $d$ assets are represented by a process $(S(t))_{t=0,1, \ldots}$, $S(t)=\left(S^{1}(t), \ldots, S^{d}(t)\right) \in(0, \infty)^{d}$. Economic factors are modelled by a time homogeneous Markov process $(Z(t))_{t=0,1, \ldots}$ with values in a Polish (separable, complete, metric) space $E$ with Borel $\sigma$-algebra $\mathcal{E}$. The dynamics of the price process are governed by the equation

$$
\frac{S^{i}(t+1)}{S^{i}(t)}=\zeta^{i}(Z(t+1), \xi(t+1)), \quad S^{i}(0)=s^{i}>0, \quad i=1, \ldots, d,
$$

where $(\xi(t))_{t=1,2, \ldots}$ is a sequence of i.i.d. random variables with values in a Polish space $\left(E^{\xi}, \mathcal{E}^{\xi}\right)$ and the functions $\zeta^{i}:(E, \mathcal{E}) \times\left(E^{\xi}, \mathcal{E}^{\xi}\right) \rightarrow(0, \infty)$ are Borel measurable for $i=1, \ldots, d$. We assume that $(S(t), Z(t))$ forms a weak Feller process, i.e. its transition operator transforms the space of bounded continuous functions into itself. In the following we shall write $\zeta^{i}(t)$ for $\zeta^{i}(Z(t), \xi(t))$, and $\zeta(t)$ for the vector $\left(\zeta^{1}(t), \ldots, \zeta^{d}(t)\right)$, whenever this does not lead to ambiguity.

Let $\left(\mathcal{F}_{t}\right)$ be a filtration generated by $(S(t), Z(t))$. It represents the knowledge of an investor observing the market. Therefore, in general, it depends on the starting point of the process $(S(t), Z(t))$. Due to (3) the filtration is in fact independent of $S(0)$. It depends only on the initial value $z$ of the process $(Z(t))$, and to stress this dependence it will be denoted by $\left(\mathcal{F}_{t}^{z}\right)$.

Fix $s \in(0, \infty)^{d}$ and $z \in E$, the initial values of the processes $(S(t))$ and $(Z(t))$. A trading strategy is a sequence of pairs $\left(\left(N_{k}, \tau_{k}\right)\right)_{k=0,1, \ldots}$, where $\tau_{0}=0,\left(\tau_{k}\right)_{k=1,2, \ldots}$ are $\left(\mathcal{F}_{t}^{z}\right)$ stopping times, and $\tau_{k+1}>\tau_{k}, k=1,2, \ldots$ The stopping times $\left(\tau_{k}\right), k \geq 1$, represent times of transactions, whereas $\tau_{0}=0$ is only introduced for convenience of notation. The number of shares held in the portfolio in the time interval $\left[\tau_{k}, \tau_{k+1}\right)$ is denoted by $N_{k}$, which is an $\mathcal{F}_{\tau_{k}}^{z}$-measurable random variable with values in $[0, \infty)^{d}$. Hence, $N_{0}$ is a deterministic initial portfolio. 
The share holding process is given by

$$
N(t)=\sum_{k=1}^{\infty} 1_{t \in\left[\tau_{k}, \tau_{k+1}\right)} N_{k}, \quad t \geq 0 .
$$

In what follows we shall consider transaction costs of one of the forms

$$
\begin{aligned}
& \widetilde{c}\left(\eta_{1}, \eta_{2}, S\right)=\sum_{i=1}^{d}\left(c_{i}^{1} S^{i}\left(\eta_{1}^{i}-\eta_{2}^{i}\right)^{+}+c_{i}^{2} S^{i}\left(\eta_{1}^{i}-\eta_{2}^{i}\right)^{-}\right)+C, \\
& \widetilde{c}\left(\eta_{1}, \eta_{2}, S\right)=\max \left(C, \sum_{i=1}^{d}\left(c_{i}^{1} S^{i}\left(\eta_{1}^{i}-\eta_{2}^{i}\right)^{+}+c_{i}^{2} S^{i}\left(\eta_{1}^{i}-\eta_{2}^{i}\right)^{-}\right)\right),
\end{aligned}
$$

where $c_{i}^{1}, c_{i}^{2} \in[0,1)$ are proportional costs, $C \geq 0, S$ stands for the asset prices at the time of transaction, $\eta_{1}$ denotes the portfolio contents before transaction, and $\eta_{2}$ after transaction. We impose a self-financing condition on portfolios, i.e.

$$
N_{k} \cdot S\left(\tau_{k}\right)=N_{k-1} \cdot S\left(\tau_{k}\right)+\widetilde{c}\left(N_{k-1}, N_{k}, S\left(\tau_{k}\right)\right), \quad k=1,2, \ldots
$$

Notice that due to the lower bound $C$ on the transaction costs function, transactions cannot be executed if the wealth of the portfolio is smaller than $C$. It is also clear that (6) depends on the initial value $(s, z)$ of the process $(S(t), Z(t))$.

For the clarity of presentation, we shall restrict our attention to the costs of the form (4). However, all the results are easily modified to fit (5), and, in fact, they extend to a larger family of transaction costs structures (see Section 6).

In the case of no transaction costs or proportional transaction costs it is natural to reformulate the problem in terms of proportions as it transforms the set of controls (possible portfolios) to a compact set, which facilitates mathematical analysis. In our more general framework, we shall also benefit from this reformulation.

Denote by $X_{-}(t)$ the wealth of the portfolio before a possible transaction at $t$ and by $X(t)$ the wealth just after the transaction:

$$
X(t)=N(t) \cdot S(t), \quad X_{-}(t)=N(t-1) \cdot S(t) .
$$

If there is no transaction at $t$ the two values are identical. In a similar way, for $i=1, \ldots, d$, we construct two processes representing proportions of our capital invested in asset $i$ :

$$
\pi^{i}(t)=\frac{N^{i}(t) S^{i}(t)}{X(t)}, \quad \pi_{-}^{i}(t)=\frac{N^{i}(t-1) S^{i}(t)}{X_{-}(t)} .
$$

Since short sales are prohibited we have $\pi(t), \pi_{-}(t) \in \mathcal{S}$, where $\mathcal{S}$ is the unit 
simplex in $\mathbb{R}^{d}$ :

$$
\mathcal{S}=\left\{\left(\pi^{1}, \ldots, \pi^{d}\right): \pi^{i} \geq 0, \sum_{i=1}^{d} \pi^{i}=1\right\}
$$

Denote by $\mathcal{S}^{0}$ the polyhedral set generated by $\mathcal{S}$ :

$$
\mathcal{S}^{0}=\left\{\left(\pi^{1}, \ldots, \pi^{d}\right): \pi^{i} \geq 0, \sum_{i=1}^{d} \pi^{i} \leq 1\right\}
$$

and let $g:[0, \infty)^{d} \backslash\{0\} \rightarrow \mathcal{S}$ be a projection to $\mathcal{S}$,

$$
g\left(\pi^{1}, \ldots, \pi^{d}\right)=\left(\frac{\pi^{1}}{\sum \pi^{i}}, \ldots, \frac{\pi^{d}}{\sum \pi^{i}}\right)
$$

Define

$$
c\left(\pi_{-}, \tilde{\pi}\right)=\sum_{i=1}^{d}\left(c_{i}^{1}\left(\tilde{\pi}^{i}-\pi_{-}^{i}\right)^{+}+c_{i}^{2}\left(\tilde{\pi}^{i}-\pi_{-}^{i}\right)^{-}\right) .
$$

The self-financing condition (6) can be written as

(9) $X_{-}\left(\tau_{k}\right)=X\left(\tau_{k}\right)+X_{-}\left(\tau_{k}\right)\left(c\left(\pi_{-}\left(\tau_{k}\right), \tilde{\pi}_{k}\right)+\frac{C}{X_{-}\left(\tau_{k}\right)}\right), \quad k=1,2, \ldots$,

for some $\tilde{\pi}_{k} \in \mathcal{S}^{0}$ such that $\pi\left(\tau_{k}\right)=g\left(\tilde{\pi}_{k}\right)$. From (6) one can deduce that $\tilde{\pi}_{k}=\frac{X\left(\tau_{k}\right)}{X_{-}\left(\tau_{k}\right)} \pi\left(\tau_{k}\right)$ satisfies $(9)$.

Given $\pi_{-}, \pi \in \mathcal{S}, x_{-}>0$ define a function

$$
F^{\pi_{-}, \pi, x_{-}}(\delta)=c\left(\pi_{-}, \delta \pi\right)+\frac{C}{x_{-}}+\delta .
$$

Equation (9) can be written equivalently as

$$
F^{\pi_{-}\left(\tau_{k}\right), \pi\left(\tau_{k}\right), X_{-}\left(\tau_{k}\right)}\left(\sum_{i=1}^{d} \tilde{\pi}_{k}^{i}\right)=1 .
$$

The following lemma states a crucial property of $F$ that will be used to reformulate the self-financing condition.

LEMmA 2.1. There exists a unique function $e: \mathcal{S} \times \mathcal{S} \times(0, \infty) \rightarrow[0,1]$ such that

(1) if $e\left(\pi_{-}, \pi, x\right)>0$, then $F^{\pi_{-}, \pi, x_{-}}\left(e\left(\pi_{-}, \pi, x_{-}\right)\right)=1$,

(2) $e\left(\pi_{-}, \pi, x_{-}\right)=0$ if and only if the equation $F^{\pi_{-}, \pi, x_{-}}(\cdot)=1$ has no solution in $(0,1]$.

Moreover, e is continuous.

Proof. The proof is rather straightforward and resembles the proof of Lemma 1 in [31]. 
Let $\left(\left(N_{k}, \tau_{k}\right)\right)$ be a self-financing trading strategy and let $\pi_{-}\left(\tau_{k}\right), \pi\left(\tau_{k}\right)$ be defined as above. By virtue of Lemma 2.1 for any $k \in \mathbb{N}$ we have

$$
F^{\pi_{-}\left(\tau_{k}\right), \pi\left(\tau_{k}\right), X_{-}\left(\tau_{k}\right)}\left(e\left(\pi_{-}\left(\tau_{k}\right), \pi\left(\tau_{k}\right), X_{-}\left(\tau_{k}\right)\right)\right)=1
$$

and $X\left(\tau_{k}\right) / X_{-}\left(\tau_{k}\right)=e\left(\pi_{-}\left(\tau_{k}\right), \pi\left(\tau_{k}\right), X_{-}\left(\tau_{k}\right)\right)$. The second assertion is a consequence of the uniqueness of $e$ and equation (9). Therefore, any transaction can be described solely by means of the proportions $\pi_{-}\left(\tau_{k}\right)$ and $\pi\left(\tau_{k}\right)$, and the portfolio wealth $X_{-}\left(\tau_{k}\right)$. Consequently, any trading strategy has a unique representation in the following form: the initial wealth $x_{-}=N_{0} \cdot S(0)$, the initial proportion

$$
\pi_{-}=\left(\frac{N_{0}^{1} S^{1}(0)}{N_{0} \cdot S(0)}, \ldots, \frac{N_{0}^{d} S^{d}(0)}{N_{0} \cdot S(0)}\right),
$$

and $\left(\left(\pi_{k}, \tau_{k}\right)\right)_{k=1,2, \ldots}$, where $\pi_{k}$ is the post-transaction proportion represented by an $\mathcal{S}$-valued $\mathcal{F}_{\tau_{k}}$-measurable random variable. Indeed, define the corresponding pre-transaction proportion process $\pi_{-}(t)$ by

$$
\begin{aligned}
& \pi_{-}(0)=\pi_{-}, \\
& \pi_{-}(t)=\pi_{k} \diamond \zeta\left(\tau_{k}+1\right) \diamond \cdots \diamond \zeta(t), \quad \tau_{k}<t \leq \tau_{k+1},
\end{aligned}
$$

where for simplicity of the notation we have $\tau_{0}=0$ and

$$
\pi \diamond \zeta=g\left(\pi^{1} \zeta^{1}, \ldots, \pi^{d} \zeta^{d}\right), \quad \pi \in \mathcal{S}, \quad \zeta \in(0, \infty)^{d} .
$$

The corresponding post-transaction proportion process is given by

$$
\pi(t)= \begin{cases}\pi_{-}, & t=0 \text { and } \tau_{1}>0, \\ \pi_{k}, & t=\tau_{k}, \\ \pi_{k} \diamond \zeta\left(\tau_{k}+1\right) \diamond \cdots \diamond \zeta(t), & \tau_{k}<t<\tau_{k+1} .\end{cases}
$$

At time $\tau_{k}$ the pre-transaction wealth $X_{-}\left(\tau_{k}\right)$ is diminished to

$$
X\left(\tau_{k}\right)=X_{-}\left(\tau_{k}\right) e\left(\pi_{-}\left(\tau_{k}\right), \pi\left(\tau_{k}\right), X_{-}\left(\tau_{k}\right)\right) .
$$

Furthermore,

$$
X_{-}(t+1)=\sum_{i=1}^{d} \frac{\pi^{i}(t) X(t)}{S^{i}(t)} S^{i}(t+1)=X(t)(\pi(t) \cdot \zeta(t+1)) .
$$

Consequently,

$$
=X_{-}(0) \prod_{s=0}^{t-1}(\pi(s) \cdot \zeta(s+1)) \prod_{k=1}^{\infty}\left(1_{\tau_{k}<t} e\left(\pi_{-}\left(\tau_{k}\right), \pi\left(\tau_{k}\right), X_{-}\left(\tau_{k}\right)\right)+1_{\tau_{k} \geq t}\right),
$$

which finishes the construction of the correspondence between the primal definition of a trading strategy with the share holding process $N(t)$ and the equivalent form with proportions. Notice that due to our reformulation, the 
self-financing condition no longer depends on the initial value of the asset price process $(S(t))$.

Let $\mathcal{A}^{z}$ be the set of sequences $\left(\left(\pi_{k}, \tau_{k}\right)\right)_{k=1,2, \ldots}$, where $\tau_{k}$ is an $\left(\mathcal{F}_{t}^{z}\right)$ stopping time, $\pi_{k}$ is an $\mathcal{F}_{\tau_{k}}^{z}$-measurable random variable with values in $\mathcal{S}$, and $\tau_{k+1}>\tau_{k}, k=1,2, \ldots$ Elements of $\mathcal{A}^{z}$ will be called admissible trading strategies or admissible portfolios. Notice that for a fixed initial wealth $x_{-}$ and an initial proportion $\pi_{-}$not every admissible trading strategy $\left(\left(\pi_{k}, \tau_{k}\right)\right)$ corresponds to some self-financing strategy $\left(\left(N_{k}, \tau_{k}\right)\right)$. Indeed, if $X_{-}\left(\tau_{k}\right)$ is small for some $k$, not all proportions $\pi\left(\tau_{k}\right)$ are attainable from $\pi_{-}\left(\tau_{k}\right)$. If $\pi\left(\tau_{k}\right)$ is attainable, we have

and

$$
F^{\pi_{-}\left(\tau_{k}\right), \pi\left(\tau_{k}\right), X_{-}\left(\tau_{k}\right)}\left(e\left(\pi_{-}\left(\tau_{k}\right), \pi\left(\tau_{k}\right), X_{-}\left(\tau_{k}\right)\right)\right)=1
$$

$$
X\left(\tau_{k}\right)=X_{-}\left(\tau_{k}\right) e\left(\pi_{-}\left(\tau_{k}\right), \pi\left(\tau_{k}\right), X_{-}\left(\tau_{k}\right)\right) .
$$

If $\pi\left(\tau_{k}\right)$ is not attainable, we have, by the above construction,

$$
e\left(\pi_{-}\left(\tau_{k}\right), \pi\left(\tau_{k}\right), X_{-}\left(\tau_{k}\right)\right)=0
$$

and

$$
X\left(\tau_{k}\right)=0 .
$$

Therefore, in what follows we may assume that all proportions are attainable from $\pi_{-}\left(\tau_{k}\right)$ irrespective of the value of $X_{-}\left(\tau_{k}\right)$, but they may lead to a zero wealth process if we cannot afford to pay transaction costs. Since the strategy allowing annihilation of wealth is not optimal (the functional in (2) evaluates to $-\infty$ ), the extension of the set of trading strategies does not have any impact on optimal strategies.

As noticed before, the set of admissible strategies and the wealth of the portfolio are independent of the initial prices of the assets. Therefore, instead of writing $\mathbb{P}^{(s, z)}$ and $\mathbb{E}^{(s, z)}$ to stress dependence of the probability measure on the initial state of the Markov process $(S(t), Z(t))$ we will write $\mathbb{P}^{z}$ and $\mathbb{E}^{z}$.

The goal of this paper is to maximize the functional

$$
J^{\pi_{-}, x_{-}, z}(\Pi)=\liminf _{T \rightarrow \infty} \frac{1}{T} \mathbb{E}^{z} \ln X_{-}(T)
$$

over all portfolios $\Pi \in \mathcal{A}^{z}$, where $\pi_{-}$is the initial proportion, $x_{-}$denotes the initial wealth and $z$ is the initial state of the economic factor process. Observe that using (13) we obtain

$$
\begin{aligned}
J^{\pi_{-}, x_{-}, z}(\Pi)=\liminf _{T \rightarrow \infty} \frac{1}{T}\{ & \sum_{t=0}^{T-1} \mathbb{E}^{z} \ln \pi(t) \cdot \zeta(t+1) \\
& \left.+\sum_{k=1}^{\infty} \mathbb{E}^{z}\left\{1_{\tau_{k}<T} \ln e\left(\pi_{-}\left(\tau_{k}\right), \pi_{k}, X_{-}\left(\tau_{k}\right)\right)\right\}\right\} .
\end{aligned}
$$

This transforms our problem to a form suitable for further analysis. 
3. Assumptions and basic properties of the price process. Denote by $P(z, d y)$ the transition operator of the process $(Z(t))$. Let $\widehat{E}=E \times E^{\xi}$, and let $\nu$ be the law of $\xi(1)$ on $E^{\xi}$. For $x=(z, \xi)$ and a bounded measurable function $w$ on $\widehat{E}$ define

$$
\widehat{P} w(x)=\int_{E} \int_{E^{\xi}} w\left(z^{\prime}, \xi^{\prime}\right) \nu\left(d \xi^{\prime}\right) P\left(z, d z^{\prime}\right) .
$$

Consider the following assumptions:

(A1) The process $(S(t), Z(t))$ has the Feller property, i.e. its transition operator maps the space of continuous bounded functions into itself.

(A2) $\mathcal{S} \times E \ni(\pi, z) \mapsto h(\pi, z)=\mathbb{E}^{z}\{\ln \pi \cdot \zeta(Z(1), \xi(1))\}$ is a bounded, continuous function.

(A3) $\quad \sup \sup \left(P^{n}(z, B)-P^{n}\left(z^{\prime}, B\right)\right)=\kappa<1$ for some $n \geq 1$. $z, z^{\prime} \in E B \in \mathcal{E}$

(A4) There is a continuous function $\widehat{u}_{0}$ defined on $\widehat{E}$ such that $\widehat{u}_{0}(x) \geq 1$ for $x \in \widehat{E}$, the function $x \mapsto \widehat{P} \widehat{u}_{0}(x)$ is bounded on compact subsets of $\widehat{E}$ and for any positive real number $l$ the set $\left\{x: \widehat{u}_{0}(x) / \widehat{P} \widehat{u}_{0}(x)\right.$ $\leq l\}$ is compact.

(A5) The function $\zeta(z, \xi)$ is continuous and bounded away from 0, i.e. $\inf _{z, \xi} \zeta^{i}(z, \xi)>0$ for $i=1, \ldots, d$.

Due to assumption (A3) the process $Z(t)$ is uniformly ergodic. Together with (A4)-(A5) this gives important estimates on the behavior of the asset prices, as can be seen in the following theorem:

THEOREM 3.1. Under (A1)-(A5):

(i) The process $Z(t)$ has a unique invariant probability measure $\vartheta$.

(ii) For each nonnegative measurable function $f$ with $\int_{E} f(z) \vartheta(d z)<\infty$,

$$
\lim _{T \rightarrow \infty} \frac{1}{T} \sum_{t=0}^{T} \mathbb{E}^{z} f(Z(t))=\int_{E} f(z) \vartheta(d z) .
$$

(iii) The following large deviations estimate holds: for each $\varepsilon>0$ there exist $T^{*}, \gamma, K>0$ such that for all $T \geq T^{*}$,

$$
\mathbb{P}^{z}\left\{\frac{1}{T} \ln \left(\prod_{t=0}^{T-1} \widehat{\zeta}(Z(t+1), \xi(t+1))\right) \leq \widehat{p}-\varepsilon\right\} \leq K e^{-\gamma T}
$$

where

$$
\begin{aligned}
\widehat{\zeta}(z, \xi) & =\min \left(\zeta^{1}(z, \xi), \ldots, \zeta^{d}(z, \xi)\right) \\
\widehat{p} & =\int_{E \times E^{\xi}} \ln \widehat{\zeta}(z, \xi) \vartheta(d z) \nu(d \xi)
\end{aligned}
$$


Proof. Notice that (A3) implies that for arbitrary $z, z^{\prime} \in E$ and $B \in \mathcal{E}$,

$$
P^{n}(z, B) \leq \kappa+P^{n}\left(z^{\prime}, B\right) .
$$

Therefore, Condition (D) (a version of Doeblin's hypothesis) in $[9$, Section V.5] holds with $\phi(B)=P^{n}\left(z^{\prime}, B\right)$ for some $z^{\prime} \in E$ and $\varepsilon=(1-\kappa) / 2$. Applying (A3) one also finds that for any bounded measurable function $f$ we have

$$
\mathbb{E}^{z_{1}} f(Z(n))-\mathbb{E}^{z_{2}} f(Z(n))=\int_{E} f(z)\left(P^{n}\left(z_{1}, d z\right)-P^{n}\left(z_{2}, d z\right)\right) \leq \kappa\|f\|_{\infty} .
$$

Therefore, due to Theorems V.5.7 and V.6.2 in [9] there is a unique invariant probability measure for $(Z(t))$ and (ii) holds.

Statement (iii) results from application of large deviations theory to the Markov process $(Z(t), \xi(t))$. Recall that the transition operator of this process is denoted by $\widehat{P}$. The measure $\vartheta \otimes \nu$ is the unique invariant measure of $\widehat{P}$. Let

$$
L_{n}=\frac{1}{n} \sum_{t=1}^{n} \delta_{(Z(t), \xi(t))}, \quad n=1,2, \ldots,
$$

denote the empirical distribution of the process $(Z(t), \xi(t))$. Notice that $L_{n}$ takes values in the space $\mathcal{P}=\mathcal{P}\left(E \times E^{\xi}\right)$ of probability measures on $E \times E^{\xi}$ with the weak convergence topology. By Section 4 of [8] (see also [11] and [23]) there exists a convex lower semicontinuous function $J: \mathcal{P} \rightarrow \mathbb{R}$ (called a good rate function) such that for any compact set $\Gamma \in \mathcal{B}(\mathcal{P})$ we have

$$
\limsup _{n \rightarrow \infty} \frac{1}{n} \log \left(\sup _{z \in E} \mathbb{P}^{z}\left(\left\{\omega: L_{n}(\omega) \in \Gamma\right\}\right)\right) \leq-\inf _{\mu \in \Gamma} J(\mu) .
$$

Under assumption (A4) the above inequality holds for any closed set $\Gamma$ (not necessarily compact). By Lemma 4.2 of [8], the set of measures $\mu \in \mathcal{P}$ such that $J(\mu) \leq l$ is compact for each $l \in \mathbb{R}$. Consequently, for a closed set $\Gamma \subset \mathcal{P}$ such that $\vartheta \otimes \nu \notin \Gamma$ we have (see Proposition 1 of [11]) $\inf _{\mu \in \Gamma} J(\mu)>0$.

Define

$$
\widehat{\Gamma}=\left\{\mu \in \mathcal{P}: \int_{E \times E^{\xi}} \ln \widehat{\zeta}(z, \xi) \mu(d z \times d \xi) \leq \widehat{p}-\varepsilon\right\} .
$$

To complete the proof it is enough to show that $\inf _{\mu \in \widehat{\Gamma}} J(\mu)>0$. Due to unboundedness of $\widehat{\zeta}$ the set $\widehat{\Gamma}$ may not be closed in $\mathcal{P}$. However, under (A5), for every $N>0$,

$$
\Gamma_{N}=\left\{\mu \in \mathcal{P}: \int_{E \times E^{\xi}} \min (\ln \widehat{\zeta}(z, \xi), N) \mu(d z \times d \xi) \leq \widehat{p}-\varepsilon\right\}
$$

is closed and $\widehat{\Gamma} \subseteq \Gamma_{N}$. By the monotone convergence theorem there exists $N$ such that $\vartheta \otimes \nu \notin \Gamma_{N}$, and consequently $\inf _{\mu \in \widehat{\Gamma}_{N}} J(\mu)>0$. 
Statement (iii) of the above lemma reads that whenever the average onestep growth rate of the asset prices $\widehat{p}$ is positive then the prices grow exponentially fast on a large subset of $\Omega$, i.e. for $T>T^{*}$,

$$
\mathbb{P}\left\{S^{i}(T) \geq S^{i}(0) e^{T(\widehat{p}-\varepsilon)} \quad \forall i=1, \ldots, d\right\} \geq 1-K e^{-\gamma T} .
$$

This is a surprising result, since the condition $\widehat{p}>0$ is often viewed as a prerequisite for investors to be willing to invest on the market. Therefore, promising markets offer exponential speed of growth of investors' wealth.

The following remarks explain the assumptions (A1)-(A5):

(1) Assume that $(Z(t))$ is a Feller process. If $\zeta^{i}(z, \xi), i=1, \ldots, d$, are continuous in $z$ then $(\mathrm{A} 1)$ is satisfied. Indeed, let $\phi:(0, \infty)^{d} \times E \rightarrow \mathbb{R}$ be continuous bounded. Define

$$
g(s, z, \xi)=\int_{E} \phi\left(s^{1} \zeta^{1}(\widetilde{z}, \xi), \ldots, s^{d} \zeta^{d}(\widetilde{z}, \xi), \widetilde{z}\right) P(z, d \widetilde{z})
$$

It is continuous by the Feller property of $(Z(t))$. Consequently, the mapping

$$
(s, z) \mapsto \mathbb{E}^{(s, z)} \phi(S(1), Z(1))=\int_{E^{\xi}} g(s, z, \xi) \nu(d \xi),
$$

where $\nu$ is a distribution of $\xi(1)$ on $E^{\xi}$, is continuous by the dominated convergence theorem, and (A1) holds. In particular, if $(Z(t))$ is a Markov chain with a finite state space, (A1) is always satisfied.

(2) Assumption (A2) reads that the expected one-period growth rate is finite.

(3) Assume that $\zeta^{i}(z, \xi), i=1, \ldots, d$, are bounded functions bounded away from 0 and continuous in $z$. Clearly, $h(\pi, z)$ is bounded. By (A1), $(Z(t))$ is a Feller process, hence $h(\pi, z)$ is continuous and (A2) holds.

(4) By Jensen's inequality,

$$
\inf _{\pi \in \mathcal{S}} h(z, \pi)=\min _{i=1, \ldots, d} \mathbb{E}^{z}\left\{\ln \zeta^{i}(Z(1), \xi(1))\right\} .
$$

Therefore, $h(\pi, z)$ is bounded from below if and only if

$$
\inf _{z \in E} \mathbb{E}^{z}\left\{\ln \zeta^{i}(Z(1), \xi(1))\right\}>-\infty, \quad i=1, \ldots, d .
$$

(5) Condition (A2) does not imply boundedness of $\zeta^{i}$. Consider a generalized Black-Scholes model with economic factors (see [5], [6], [26]), i.e.

$$
\begin{array}{r}
S^{i}(t+1)=S^{i}(t) \exp \left(\sigma^{i}(Z(t+1)) \cdot(W(t+1)-W(t))+\mu^{i}(Z(t+1))\right) \\
i=1, \ldots, d
\end{array}
$$

where $(Z(t))$ is a Feller process, $(W(t))$ is an $m$-dimensional Wiener process and $\sigma^{i}: E \rightarrow \mathbb{R}^{m}, \mu^{i}: E \rightarrow \mathbb{R}, i=1, \ldots, d$, are continuous bounded func- 
tions. Clearly, (A1) is satisfied by (1). To show (A2) we recall the definition

$$
h(\pi, z)=\mathbb{E}^{z} \ln \left(\sum_{i=1}^{d} \pi^{i} \exp \left(\sigma^{i}(Z(1)) \cdot \xi(1)+\mu^{i}(Z(1))\right)\right)
$$

with $\xi(1)=W(1)-W(0)$. Consequently,

$$
\begin{aligned}
\mathbb{E}^{z}\left\{-D_{1}(Z(1))\|\xi(1)\|_{2}\right. & \left.-D_{2}(Z(1))\right\} \\
& \leq h(\pi, z) \leq \mathbb{E}^{z}\left\{D_{1}(Z(1))\|\xi(1)\|_{2}+D_{2}(Z(1))\right\},
\end{aligned}
$$

where $\xi$ has a standard normal distribution on $\mathbb{R}^{m}$, denoted by $\nu, D_{1}(z)=$ $\max _{i=1, \ldots, d}\left\|\sigma^{i}(z)\right\|_{2}, D_{2}(z)=\max _{i=1, \ldots, d}\left|\mu^{i}(z)\right|$, and $\|\cdot\|_{2}$ stands for the $L^{2}$ norm in $\mathbb{R}^{m}$. Therefore, $h(\pi, z)$ is bounded. Continuity follows from the dominated convergence theorem.

(6) In the stochastic control literature, one-step uniform ergodicity is usually assumed, i.e. (A3) with $n=1$ (see e.g. condition (UE) in [31]). Allowing for $n>1$ opens a new class of applications. In particular, if $Z(t)$ is a recursive Markov chain on a finite state space then (A3) holds with some $n>0$, but usually it does not hold with $n=1$.

Consider now the following assumption:

$\left(\mathrm{H}^{*}\right) \quad$ There is a continuous function $u_{0}$ defined on $E$ such that $u_{0}(x) \geq 1$ for $x \in E, P u_{0}(x)$ is bounded on compact subsets of $E$ and for any $l$ the set $\left\{z: u_{0}(z) / P u_{0}(z) \leq l\right\}$ is compact.

We have

LEMMA 3.2. If $E^{\xi}$ is locally compact and $\left(\mathrm{H}^{*}\right)$ is satisfied then (A4) holds.

Proof. Without loss of generality we may assume that the support of $\nu$ is not compact (otherwise we can replace $E^{\xi}$ by a compact set). Let $\left(K_{n}\right)$ be an increasing sequence of compact sets such that $\nu\left(K_{n+1} \backslash K_{n}\right) \leq 1 / n^{2}$ and $\overline{K_{n+1} \backslash K_{n}} \cap K_{n-1}=\emptyset$ for $n=1,2, \ldots$, and $\bigcup_{n} K_{n}=E^{\xi}$. Define a function $g$ on $E^{\xi}$ to be equal to 1 on $K_{1}$ and $\sqrt{n}$ on $\overline{K_{n+1} \backslash K_{n}}$ for odd $n$, and extend $g$ using the Tietze theorem to a continuous function on the whole $E^{\xi}$. The construction in the Tietze theorem implies that $g(\xi) \geq 1$ and $\nu(g):=\int_{E^{\xi}} g(\xi) \nu(d \xi)<\infty$. Let $\widehat{u}_{0}(z, \xi)=u_{0}(z) g(\xi)$. We shall prove that the set

$$
\Gamma_{l}=\left\{x \in \widehat{E}: \frac{\widehat{u}_{0}(x)}{\widehat{P} \widehat{u}_{0}(x)} \leq l\right\}=\left\{(z, \xi) \in \widehat{E}: \frac{u_{0}(z) g(\xi)}{P u_{0}(z) \nu(g)} \leq l\right\}
$$

is compact for any $l$. Let $\left(z_{n}, \xi_{n}\right) \subset \Gamma_{l}$. If $\left(\xi_{n}\right)$ leaves all compact sets $K_{m}$ then $g\left(\xi_{n}\right) \rightarrow \infty$. Consequently, $u_{0}\left(z_{n}\right) / P u_{0}\left(z_{n}\right) \rightarrow 0$, which contradicts $\inf _{z \in E} u_{0}(z) / P u_{0}(z)>0$. Therefore, there exists $m$ such that $\left(\xi_{n}\right)$ is contained in $K_{m}$. Compactness of $K_{m}$ implies that $\xi_{n_{k}} \rightarrow \xi \in K_{m}$ for some 
subsequence $n_{k}$. Since

$$
\frac{u_{0}\left(z_{n_{k}}\right)}{P u_{0}\left(z_{n_{k}}\right)} \leq \frac{l \nu(g)}{g\left(\xi_{n_{k}}\right)} \leq l \nu(g),
$$

by $\left(\mathrm{H}^{*}\right)$ there is a subsequence of $z_{n_{k}}$ convergent to $z$. By continuity of $\widehat{u}_{0} / \widehat{P} \widehat{u}_{0}$, we have $(z, \xi) \in \Gamma_{l}$, which completes the proof of compactness of $\Gamma_{l}$.

4. Discounted functionals and estimates. This section is devoted to an in-depth study of the discounted functional related to the functional (15). It plays a major role in the derivation of the Bellman inequality for our optimization problem.

Given $\pi_{-}, x_{-}, z$ consider a discounted functional

$$
=\mathbb{E}^{z}\left\{\sum_{t=0}^{\infty} \beta^{t} h(\pi(t), Z(t))+\sum_{k=1}^{\infty} \beta^{\tau_{k}} \ln e\left(\pi_{-}\left(\tau_{k}\right), \pi_{k}, X_{-}\left(\tau_{k}\right)\right)\right\}, \quad \beta \in(0,1),
$$

and its value function

$$
v_{\beta}\left(\pi_{-}, x_{-}, z\right)=\sup _{\Pi \in \mathcal{A}^{z}} J_{\beta}^{\pi_{-}, x_{-}, z}(\Pi) .
$$

Denote by $M$ an impulse operator acting on measurable functions,

$$
M w\left(\pi_{-}, x_{-}, z\right)=\sup _{\pi \in \mathcal{S}}\left\{\ln e\left(\pi_{-}, \pi, x_{-}\right)+w\left(\pi, x_{-} e\left(\pi_{-}, \pi, x_{-}\right), z\right)\right\} .
$$

LEMma 4.1. The impulse operator maps the space of continuous bounded functions into itself. Moreover, given any bounded continuous function $w$ there exists a measurable selector for $M w$.

Proof. The proof is standard (see [7, Corollary 1] or [15]).

THEOREM 4.2. Under (A1)-(A2) the function $v_{\beta}$ is continuous and bounded, and satisfies the Bellman equation

$$
\begin{aligned}
& v_{\beta}\left(\pi_{-}, x_{-}, z\right) \\
& =\sup _{\tau} \mathbb{E}^{z}\left\{\sum_{t=0}^{\tau-1} \beta^{t} h(\pi(t), Z(t))+\beta^{\tau} M v_{\beta}\left(\pi_{-}(\tau), X_{-}(\tau), Z(\tau)\right)\right\},
\end{aligned}
$$

where

$$
\begin{aligned}
\pi_{-}(0) & =\pi_{-}, & \pi_{-}(t+1) & =\pi_{-}(t) \diamond \zeta(t+1), \\
X_{-}(0) & =x_{-}, & X_{-}(t+1) & =X_{-}(t)\left(\pi_{-}(t) \cdot \zeta(t+1)\right)
\end{aligned}
$$

are counterparts of (10), (12), (13).

Proof. By Lemma 2.1 the function $\ln e\left(\pi_{-}, \pi, x_{-}\right)$is bounded, and by (A2), $h(\pi, z)$ is bounded. Therefore, $v_{\beta}\left(\pi_{-}, x_{-}, z\right)$ is bounded. For a contin- 
uous bounded function $v: \mathcal{S} \times(0, \infty) \times E \rightarrow \mathbb{R}$ let

$$
\mathcal{T}_{\beta} v(\pi, x, z)=\sup _{\tau} \mathbb{E}^{z}\left\{\sum_{t=0}^{\tau-1} \beta^{t} h(\pi(t), Z(t))+\beta^{\tau} M v\left(\pi_{-}(\tau), X_{-}(\tau), Z(\tau)\right)\right\} .
$$

The operator $\mathcal{T}_{\beta}$ maps the space $C^{b}=C^{b}(\mathcal{S} \times(0, \infty) \times E ; \mathbb{R})$ of bounded continuous functions into itself. This results from the Feller property (A1) of the transition operator of the process $(S(t), Z(t))$ by a general result on the continuity of the value function of optimal stopping problems (see [30]). Let

$$
v_{\beta}^{0}\left(\pi_{-}, x_{-}, z\right)=\sum_{t=0}^{\infty} \beta^{t} \mathbb{E}^{z} h\left(\pi_{-}(t), X_{-}(t)\right) .
$$

Put $v_{\beta}^{k+1}=\mathcal{T}_{\beta} v_{\beta}^{k}$. Thanks to continuity of $v_{\beta}^{k}$ and $M v_{\beta}^{k}$ it can be shown that $v_{\beta}^{k}$ is a value function for the maximization of $J_{\beta}$ over portfolios with at most $k$ transactions. Observe that it is never optimal to have two transactions at the same time $\left(\mathbb{P}\left(\tau_{k}=\tau_{k+1}\right)>0\right)$, by the subadditivity of the transaction costs structure. Therefore, we have the estimate

$$
\left\|v_{\beta}-v_{\beta}^{k}\right\|_{\infty} \leq \sum_{l=k}^{\infty} \beta^{l}\|h\|_{\infty}=\beta^{k} \frac{\|h\|_{\infty}}{1-\beta},
$$

which implies that $v_{\beta}^{k}$ tends uniformly to $v_{\beta}$. Consequently, $v_{\beta}$ is a continuous bounded function and satisfies $v_{\beta}=\mathcal{T}_{\beta} v_{\beta}$, which is equivalent to the Bellman equation (18).

There are two distinct cases: $C>0$ (fixed plus proportional transaction costs) and $C=0$ (proportional costs only). Theorem 4.2 applies to both. The rest of this section is devoted to estimation of the difference between value functions of problems with and without a fixed term in transaction costs. Let us first examine the equation for $e\left(\pi_{-}, \pi, x_{-}\right)$:

$$
c\left(\pi_{-}, e\left(\pi_{-}, \pi, x_{-}\right) \pi\right)+\frac{C}{x_{-}}+e\left(\pi_{-}, \pi, x_{-}\right)=1 .
$$

Clearly, if $C=0$, the solution is independent of $x_{-}$. Similarly, for $C=0$, the impulse operator $M$ does not depend on $x_{-}$, hence the value function $v_{\beta}$ is independent of $x_{-}$. Therefore, in the case without a fixed term in transaction costs, we shall skip $x_{-}$in the list of arguments and write $\widetilde{J}_{\beta}^{\pi_{-}, z}(\Pi), \widetilde{v}_{\beta}\left(\pi_{-}, z\right)$ and $\widetilde{e}\left(\pi_{-}, \pi\right)$.

4.1. Technical estimates. This subsection presents auxiliary results. They are similar to those obtained in [25]. For completeness, their proofs are included in Appendix.

Due to self-financing of portfolios, transaction costs decrease portfolio wealth. It is therefore important to derive estimates on the diminution factor 
$e\left(\pi_{-}, \pi, x_{-}\right)$and to study the relationship between $e\left(\pi_{-}, \pi, x_{-}\right)$and $\widetilde{e}\left(\pi_{-}, \pi\right)$. The first lemma below states lower bounds for $e$ and $\widetilde{e}$ :

LEMMA 4.3. We have

$$
\begin{aligned}
1-\widetilde{e}\left(\pi_{-}, \pi\right) & \leq \frac{2 \max _{i}\left(c_{i}^{1}, c_{i}^{2}\right)}{1-\max _{i}\left(c_{i}^{1}, c_{i}^{2}\right)}, \\
1-e\left(\pi_{-}, \pi, x_{-}\right) & \leq \frac{2 \max _{i}\left(c_{i}^{1}, c_{i}^{2}\right)+C / x_{-}}{1-\max _{i}\left(c_{i}^{1}, c_{i}^{2}\right)} .
\end{aligned}
$$

Let $x^{*}=\inf \left\{x_{-}: e\left(\pi_{-}, \pi, x_{-}\right)>0\right.$ for all $\left.\pi_{-}, \pi \in \mathcal{S}\right\}$. If the wealth of the portfolio is greater than $x^{*}$, any transaction can be executed. We use this rough threshold in the following lemma:

Lemma 4.4. For $\pi_{-}, \pi \in \mathcal{S}$ :

(i) $e\left(\pi_{-}, \pi, \widetilde{x}_{-}\right) \leq e\left(\pi_{-}, \pi, x_{-}\right) \leq \widetilde{e}\left(\pi_{-}, \pi\right), \quad x_{-} \geq \widetilde{x}_{-}>0$.

(ii) $\widetilde{e}\left(\pi_{-}, \pi\right)-e\left(\pi_{-}, \pi, x_{-}\right) \leq \frac{C}{\left(1-\max _{i} c_{i}^{1}\right) x_{-}} \quad$ for $x_{-}>x^{*}$.

(iii) For all $M>x^{*}$ and $x_{-} \geq M$,

$$
\ln \frac{\widetilde{e}\left(\pi_{-}, \pi\right)}{e\left(\pi_{-}, \pi, x_{-}\right)} \leq \frac{1}{\inf _{\widetilde{\pi}_{-}, \tilde{\pi}} e\left(\widetilde{\pi}_{-}, \widetilde{\pi}, M\right)} \frac{C}{\left(1-\max _{i} c_{i}^{1}\right) x_{-}} .
$$

COROllary 4.5. The value function $v_{\beta}\left(\pi_{-}, x_{-}, z\right)$ is nondecreasing in $x_{-}$.

By Theorem 4.2 the value function $\widetilde{v}_{\beta}\left(\pi_{-}, z\right)$ is bounded and continuous for each $\beta$. However, this does not imply that it is uniformly bounded in $\beta$. On the contrary, it increases to infinity as $\beta$ grows to 1 in market models of interest. To account for this fact, we shall study the span seminorm of $\widetilde{v}_{\beta}$ defined as $\left\|\widetilde{v}_{\beta}\right\|_{\text {sp }}=\sup \widetilde{v}_{\beta}(\cdot)-\inf \widetilde{v}_{\beta}(\cdot)$.

Lemma 4.6. Under (A3) there exists $M<\infty$ such that

$$
\left\|\widetilde{v}_{\beta}\right\|_{\mathrm{sp}} \leq M
$$

for all $\beta \in(0,1)$.

4.2. Large deviations and proportional transaction costs. Theorem 3.1 provides an important insight into the dynamics of asset prices. In this section we apply this result to describe the dynamics of the portfolio wealth under proportional transaction costs. Let us introduce a general assumption: (A6) $\eta<\widehat{p}$,

where the $\widehat{p}$ is the constant from Theorem 3.1 and

$$
\eta=-\ln \left(1-\frac{2 \max _{i}\left(c_{i}^{1}, c_{i}^{2}\right)}{1-\max _{i}\left(c_{i}^{1}, c_{i}^{2}\right)}\right) .
$$


Since $\eta$ is a unique solution to the equation

$$
e^{-\eta}=1-\frac{2 \max _{i}\left(c_{i}^{1}, c_{i}^{2}\right)}{1-\max _{i}\left(c_{i}^{1}, c_{i}^{2}\right)},
$$

by virtue of Lemma $4.3, e^{-\eta}$ is a lower bound on $\widetilde{e}\left(\pi_{-}, \pi\right)$. Formula (13) gives the following estimate:

$$
X_{-}(t) \geq X_{-}(0) \prod_{s=0}^{t-1}\left(e^{-\eta} \pi(s) \cdot \zeta(s+1)\right)=X_{-}(0) e^{-\eta t} \prod_{s=0}^{t-1}(\pi(s) \cdot \zeta(s+1)) .
$$

Consequently, setting $\widehat{\zeta}(t)=\min \left(\zeta^{1}(t), \ldots, \zeta^{d}(t)\right)$, we obtain

$$
X_{-}(t) \geq X_{-}(0) e^{-\eta t} \prod_{s=0}^{t-1} \widehat{\zeta}(s+1)
$$

In view of Theorem 3.1 for any $\varepsilon>0$ there exist $K, \gamma>0$ and $T^{*}$ such that

$$
\mathbb{P}\left\{e^{T(\widehat{p}-\varepsilon)} \leq \prod_{s=0}^{t-1} \widehat{\zeta}(s+1)\right\} \geq 1-K e^{-\gamma T}, \quad T>T^{*},
$$

and the wealth of the portfolio satisfies

$$
\mathbb{P}\left\{X_{-}(0) e^{T(\widehat{p}-\eta-\varepsilon)} \leq X_{-}(T)\right\} \geq 1-K e^{-\gamma T}, \quad T>T^{*} .
$$

Due to (A6) there exists an $\varepsilon$ with $0<\varepsilon<\widehat{p}-\eta$, which implies that $X_{-}(t)$ increases exponentially fast irrespective of the portfolio trading strategy.

4.3. Bounds on $\widetilde{v}_{\beta}\left(\pi_{-}, z\right)-v_{\beta}\left(\pi_{-}, x_{-}, z\right)$. For the rest of this section assume that (A1)-(A6) are satisfied. Since

$$
\lim _{m \rightarrow \infty}-\ln \left(1-\frac{2 \max _{i}\left(c_{i}^{1}, c_{i}^{2}\right)+C / m}{1-\max _{i}\left(c_{i}^{1}, c_{i}^{2}\right)}\right)=\eta<\widehat{p},
$$

there exists a constant $M>0$ such that

$$
\widehat{p}>\eta_{M}:=-\ln \left(1-\frac{2 \max _{i}\left(c_{i}^{1}, c_{i}^{2}\right)+C / M}{1-\max _{i}\left(c_{i}^{1}, c_{i}^{2}\right)}\right) .
$$

THEOREM 4.7. For any $z \in E$ and any admissible strategy $\widetilde{\Pi} \in \mathcal{A}^{z}$, $\pi_{-} \in \mathcal{S}, x_{-} \in(0, \infty)$ there exists an admissible trading strategy $\Pi \in \mathcal{A}^{z}$ such that

$$
\widetilde{J}_{\beta}^{\pi_{-}, z}(\widetilde{\Pi})-J_{\beta}^{\pi_{-}, x_{-}, z}(\Pi) \leq L\left(x_{-}\right), \quad \beta \in(0,1),
$$

where

$$
L\left(x_{-}\right)=K_{1}+K_{2} \max \left(K_{3},-\ln x_{-}\right)
$$

for some strictly positive constants $K_{1}, K_{2}, K_{3}$ independent of the choice of $z, \widetilde{\Pi}, \pi_{-}$and $x_{-}$. 
Corollary 4.8. We have

$$
0 \leq \widetilde{v}_{\beta}\left(\pi_{-}, z\right)-v_{\beta}\left(\pi_{-}, x_{-}, z\right) \leq L\left(x_{-}\right), \quad \beta \in(0,1), \pi_{-} \in \mathcal{S}, z \in E .
$$

where $L\left(x_{-}\right)$is the function from Theorem 4.7 .

Proof. The inequality $0 \leq \widetilde{v}_{\beta}\left(\pi_{-}, z\right)-v_{\beta}\left(\pi_{-}, x_{-}, z\right)$ is obvious. For the second inequality it is enough to notice that

$$
\widetilde{v}_{\beta}\left(\pi_{-}, z\right)-v_{\beta}\left(\pi_{-}, x_{-}, z\right) \leq \sup _{\widetilde{\Pi} \in \mathcal{A}}\left\{\widetilde{J}_{\beta}^{\pi_{-}, z}(\widetilde{\Pi})-\widetilde{J}_{\beta}^{\pi_{-}, x_{-}, z}(\Pi)\right\},
$$

where $\Pi$ denotes a strategy related to $\widetilde{\Pi}$ as in Theorem 4.7 .

The strength of the above theorem and corollary lies in the fact that the estimates are uniform in $\beta, \pi_{-}$and $z$.

Proof of Theorem 4.7. Fix $\pi_{-}, x_{-}, z$ and $\widetilde{\Pi} \in \mathcal{A}^{z}$. We construct $\Pi$, with its pre-transaction wealth denoted by $X_{-}(t)$, in the following way: if $X_{-}(t) \geq$ $M$ we mimic the strategy $\widetilde{\Pi}$, i.e. we keep the same proportions of stocks. On the other hand, if $X_{-}(t)$ is smaller than $M$ we do not make any transactions and wait for the wealth to raise over $M^{*}=M e^{\eta_{M}}$. At that time we perform a transaction to make the proportions equal to those defined by $\widetilde{\Pi}$. This decreases the wealth at most by $e^{-\eta_{M}}$, so the resulting portfolio wealth is not less than $M$.

Let $\tilde{\pi}_{-}(t)$ and $\tilde{\pi}(t)$ denote the pre-transaction and post-transaction processes corresponding to the strategy $\widetilde{\Pi}$. Analogously, $\pi_{-}(t)$ and $\pi(t)$ are the processes corresponding to $\Pi$. By the construction of $\Pi$ we know that $\pi(t)=\tilde{\pi}(t)$ if $X_{-}(t) \geq M^{*}$. However, if the wealth $X_{-}(t)$ is below $M^{*}$ but above $M$ we cannot determine whether $\pi(t)=\widetilde{\pi}(t)$. This is caused by the fact that the wealth $X_{-}(t)$ can be between $M$ and $M^{*}$ as a result of either a normal investing process or recovering from the shortage of wealth (being below $M)$.

By the definition of $J_{\beta}^{\pi_{-}, x_{-}, z}$ and $\widetilde{J}_{\beta}^{\pi_{-}, z}$ we have

$$
\begin{aligned}
\widetilde{J}_{\beta}^{\pi_{-}, z}(\widetilde{\Pi})-J_{\beta}^{\pi_{-}, x_{-}, z}(\Pi) & =\mathbb{E}^{z}\left\{\sum_{t=0}^{\infty} \beta^{t}(h(\widetilde{\pi}(t), Z(t))-h(\pi(t), Z(t))\right. \\
& \left.\left.+1_{\widetilde{\pi}_{-}(t) \neq \widetilde{\pi}(t)} \ln \widetilde{e}\left(\widetilde{\pi}_{-}(t), \widetilde{\pi}(t)\right)-1_{\pi_{-}(t) \neq \pi(t)} \ln e\left(\pi_{-}(t), \pi(t), X_{-}(t)\right)\right)\right\} .
\end{aligned}
$$

The above difference can be bounded from above by the sum of the following two expressions:

$$
\begin{aligned}
\mathbb{E}^{z}\left\{\sum_{t=0}^{\infty} \beta^{t} 1_{X_{-}(t)<M^{*}}(h(\tilde{\pi}(t),\right. & Z(t))-h(\pi(t), Z(t)) \\
& \left.\left.-1_{\pi_{-}(t) \neq \pi(t)} \ln e\left(\pi_{-}(t), \pi(t), X_{-}(t)\right)\right)\right\}
\end{aligned}
$$




$$
\begin{aligned}
\mathbb{E}^{z}\left\{\sum_{t=0}^{\infty} \beta^{t} 1_{X_{-}(t) \geq M^{*}}(h(\widetilde{\pi}(t),\right. & Z(t))-h(\pi(t), Z(t)) \\
& \left.\left.+1_{\pi_{-}(t) \neq \pi(t)} \ln \frac{\widetilde{e}\left(\pi_{-}(t), \pi(t)\right)}{e\left(\pi_{-}(t), \pi(t), X_{-}(t)\right)}\right)\right\} .
\end{aligned}
$$

By construction of the strategy $\Pi$ no transaction is performed if $X_{-}(t)$ $<M$, so

$$
-1_{\pi_{-}(t) \neq \pi(t)} \ln e\left(\pi_{-}(t), \pi(t), X_{-}(t)\right) \leq \eta_{M} .
$$

This yields

$$
(21) \leq L_{1} \mathbb{E}^{z} \sum_{t=0}^{\infty} 1_{X_{-}(t)<M^{*}},
$$

where $L_{1}=\sup h(\cdot)-\inf h(\cdot)+\eta_{M}$. On the other hand, if $X_{-}(t) \geq M^{*}$, we have $\pi(t)=\tilde{\pi}(t)$, so

$$
(22) \leq \mathbb{E}^{z}\left\{\sum_{t=0}^{\infty}\left(1_{X_{-}(t) \geq M^{*}} \ln \frac{\widetilde{e}\left(\pi_{-}(t), \pi(t)\right)}{e\left(\pi_{-}(t), \pi(t), X_{-}(t)\right)}\right)\right\} .
$$

By virtue of Lemma 4.4(iii) we obtain

$$
(22) \leq \mathbb{E}^{z}\left\{\sum_{t=0}^{\infty}\left(1_{X_{-}(t) \geq M^{*}} \frac{L_{2}}{X_{-}(t)}\right)\right\},
$$

where

$$
L_{2}=\frac{C}{\inf _{\widehat{\pi}_{-}, \widehat{\pi}} e\left(\widehat{\pi}_{-}, \widehat{\pi}, M^{*}\right)} .
$$

Consequently, we have the estimate

$$
\begin{aligned}
& \widetilde{J}_{\beta}^{\pi_{-}, z}(\widetilde{\Pi})-J_{\beta}^{\pi_{-}, x_{-}, z}(\Pi) \\
& \leq L_{1} \mathbb{E}^{z} \sum_{t=0}^{\infty} 1_{X_{-}(t)<M^{*}}+L_{2} \mathbb{E}^{z} \sum_{t=0}^{\infty}\left(1_{X_{-}(t) \geq M^{*}} \frac{1}{X_{-}(t)}\right) .
\end{aligned}
$$

To complete the proof we use the large deviations estimate. Fix $\varepsilon>0$ small enough that $\widehat{p}-\eta_{M}-\varepsilon>0$. Denote by $A_{t}$ the event

$$
A_{t}=\left\{\frac{1}{t} \ln \left(\prod_{j=0}^{t-1} \widehat{\zeta}(Z(j+1), \xi(j+1))\right)-\widehat{p} \geq-\varepsilon\right\} .
$$

The strategy $\Pi$ is constructed in such a way that trade takes place only if $X_{-}(t) \geq M$. Thus on the set $A_{t}$ we have

$$
X_{-}(t) \geq x_{-} e^{-t \eta_{M}} e^{t(\widehat{p}-\varepsilon)}=x_{-} e^{t\left(\widehat{p}-\eta_{M}-\varepsilon\right)} .
$$

This means an exponentially fast growth of the wealth due to $\widehat{p}-\eta_{M}-\varepsilon>0$. 
Let $K, \gamma>0$ and $T^{*}$ be the constants from Theorem 3.1(iii) for the given $\varepsilon$. By the large deviations estimate we have

$$
\mathbb{P}^{z}\left(A_{t}^{c}\right) \leq K e^{-\gamma t} \quad \text { for } t \geq T^{*},
$$

where $A_{t}^{c}$ denotes the complement of $A_{t}$. Let $t_{0}$ be the smallest integer such that $t_{0} \geq T^{*}$ and

$$
e^{t_{0}\left(\widehat{p}-\eta_{M}-\varepsilon\right)} \geq M^{*} / x_{-} .
$$

Clearly, $X_{-}(t) \geq M^{*}$ on $A_{t}$ for all $t \geq t_{0}$. Hence

$$
\mathbb{E}^{z} \sum_{t=0}^{\infty} 1_{X_{-}(t)<M^{*}} \leq t_{0}+\sum_{t=t_{0}}^{\infty} \mathbb{P}\left(A_{t}^{c}\right) \leq t_{0}+\sum_{t=t_{0}}^{\infty} K e^{-\gamma t}=: L_{3} .
$$

Computation of a bound for the second term of (23) has to be split into two parts depending on $A_{t}$ :

$$
\begin{aligned}
\mathbb{E}^{z} \sum_{t=0}^{\infty}( & \left.1_{X_{-}(t) \geq M^{*}} \frac{1}{X_{-}(t)}\right) \\
& =\mathbb{E}^{z} \sum_{t=0}^{\infty}\left(1_{X_{-}(t) \geq M^{*}} \frac{1_{A_{t}}}{X_{-}(t)}\right)+\mathbb{E}^{z} \sum_{t=0}^{\infty}\left(1_{X_{-}(t) \geq M^{*}} \frac{1_{A_{t}^{c}}}{X_{-}(t)}\right) .
\end{aligned}
$$

Clearly,

$$
\begin{aligned}
\mathbb{E}^{z} \sum_{t=0}^{\infty}( & \left.1_{X_{-}(t) \geq M^{*}} \frac{1_{A_{t}^{c}}}{X_{-}(t)}\right) \\
& \leq \frac{1}{M^{*}} \mathbb{E}^{z} \sum_{t=0}^{\infty} \mathbb{P}\left(A_{t}^{c}\right) \leq \frac{1}{M^{*}} \mathbb{E}^{z} \sum_{t=0}^{\infty} K e^{-\gamma t}=: L_{4} .
\end{aligned}
$$

By (24),

$$
\begin{aligned}
\mathbb{E}^{z} \sum_{t=0}^{\infty}( & \left.1_{X_{-}(t) \geq M^{*}} \frac{1_{A_{t}}}{X_{-}(t)}\right) \\
& \leq \mathbb{E}^{z} \sum_{t=0}^{t_{0}-1}\left(1_{X_{-}(t) \geq M^{*}} \frac{1_{A_{t}}}{X_{-}(t)}\right)+\mathbb{E}^{z} \sum_{t=t_{0}}^{\infty}\left(1_{X_{-}(t) \geq M^{*}} \frac{1_{A_{t}}}{X_{-}(t)}\right) \\
& \leq \frac{t_{0}}{M^{*}}+\frac{1}{M^{*}} \sum_{t=t_{0}}^{\infty} e^{-\left(t-t_{0}\right)\left(\widehat{p}-\eta_{M}-\varepsilon\right)}=: L_{5} .
\end{aligned}
$$

Consequently,

$$
\widetilde{J}_{\beta}^{\pi_{-}, z}(\widetilde{\Pi})-J_{\beta}^{\pi_{-}, x_{-}, z}(\Pi) \leq L_{1} L_{3}+L_{2}\left(L_{4}+L_{5}\right) .
$$

The constants $L_{1}, \ldots, L_{5}$ do not depend on $\pi_{-}, \widetilde{\Pi}$ and $z$. However, they depend on $x_{-}$through $t_{0}$. Combining the estimates for $L_{1}, \ldots, L_{5}$ we obtain the formula for $L\left(x_{-}\right)$. 
5. Growth optimal portfolios. Now we are in a position to state and prove the main result of this paper: existence and form of an optimal strategy maximizing the expected average rate of return of a portfolio of financial assets.

THEOREM 5.1. Under assumptions (A1)-(A6) there exists a measurable function $p: \mathcal{S} \times(0, \infty) \times E \rightarrow \mathcal{S}$, a constant $\lambda$ and a measurable set $I \subseteq$ $\mathcal{S} \times(0, \infty) \times E$ such that

$$
\lambda=J^{\pi_{-}, x_{-}, z}\left(\Pi^{*}\right)=\sup _{\Pi \in \mathcal{A}^{z}} J^{\pi_{-}, x_{-}, z}(\Pi),
$$

where the optimal portfolio $\Pi^{*}=\left(\left(\pi_{1}^{*}, \tau_{1}^{*}\right),\left(\pi_{2}^{*}, \tau_{2}^{*}\right), \ldots\right)$ is given by the formulas

$$
\begin{aligned}
\tau_{1}^{*} & =\inf \left\{t \geq 0:\left(\pi_{-}(t), X_{-}(t), Z(t)\right) \in I\right\}, \\
\tau_{k+1}^{*} & =\inf \left\{t>\tau_{k}^{*}:\left(\pi_{-}(t), X_{-}(t), Z(t)\right) \in I\right\}, \\
\pi_{k}^{*} & =p\left(\pi_{-}\left(\tau_{k}^{*}\right), X_{-}\left(\tau_{k}^{*}\right), Z\left(\tau_{k}^{*}\right)\right) .
\end{aligned}
$$

The strength of the above theorem is in its generality. We are not aware of papers dealing with the maximization of the average rate of return in such a general setting and with fixed and proportional transaction costs. This result also extends the area of applicability of the vanishing discount approach to models with non-weakly continuous controlled transition probabilities. Existing results require either strongly or weakly continuous (Feller) controlled transition probabilities (see [15], [19], [28], [30]). Moreover, in Section 6 we generalize Theorem 5.1 to other transaction costs structures.

Corollary 5.2.

(i) The optimal value for the problem with only proportional transaction costs $(C=0)$ is equal to the $\lambda$ from Theorem 5.1. The strategy optimal for fixed plus proportional transaction costs is also optimal for proportional transaction costs.

(ii) There exists an optimal portfolio $\Pi$ for the problem with proportional transaction costs that depends only on the current state of the processes $\left(\pi_{-}(t)\right)$ and $(Z(t))$ (and does not depend on $\left(X_{-}(t)\right)$ ).

(iii) If $\Pi$ is the portfolio from (ii), then the portfolio $\Pi_{M}$ optimal for fixed plus proportional transaction costs is constructed as follows (for notation consult Subsection 4.3): whenever $X_{-}(t)<M$, do not make any transactions and wait until the wealth increases over $M e^{\eta_{M}}$; otherwise as long as $X_{-}(t) \geq M$ keep the same proportions of stocks as in $\Pi$.

Optimal strategies for proportional costs can be efficiently computed in a number of cases: there are closed-form formulas in simple diffusion models and efficient algorithms for more complicated models, all benefiting from 
compactness of the state space. Corollary 5.2(iii) shows how an optimal portfolio for proportional costs can be employed to construct an optimal trading strategy for fixed and proportional transaction costs. However, unlike the $\Pi^{*}$ from Theorem 5.1, $\Pi_{M}$ constructed in Corollary 5.2 depends on past variations of the wealth and hence is not Markovian. A detailed proof of Corollary 5.2 is given later.

Proof of Theorem 5.1. We use a generalization of the vanishing discount method ([4], [15], [19], [28], [31]). We obtain a Bellman inequality for our optimization problem as a limit of Bellman equations for discounted problems (16). We cannot directly apply known results since they require continuity of the controlled transition function $q$ defined below. Instead, we follow the approach pioneered by [28]: we exchange the parts of argument where the continuity of $q$ is needed by considerations based on specific properties of our control problem. We also ease the requirement of local compactness of the state space in the spirit of [19].

Denote by $\mathcal{H}=\mathcal{S} \times(0, \infty) \times E$ the state space of our Markovian control model. It is complete and separable, which is needed for the existence of measurable selectors. Denote by $q$ the controlled transition operator, i.e. a function $q: \mathcal{H} \times \mathcal{S} \rightarrow \mathcal{P}(\mathcal{H})$, where $\mathcal{P}(\mathcal{H})$ is the space of Borel probability measures on $\mathcal{H}$, uniquely determined by the formula

$$
\begin{aligned}
\int_{\mathcal{H}} f\left(\widetilde{\pi}_{-}, \widetilde{x}_{-}, \widetilde{z}\right) q\left(\pi_{-}, x_{-}, z, \pi\right)\left(d \widetilde{\pi}_{-},\right. & \left.d \widetilde{x}_{-}, d \widetilde{z}\right) \\
& =\mathbb{E}^{z} f\left(\pi \diamond \zeta(z, \xi(1)), X_{-}^{\pi}(1), z(1)\right)
\end{aligned}
$$

for all bounded measurable $f: \mathcal{H} \rightarrow \mathbb{R}$, where

$$
X_{-}^{\pi}(1)= \begin{cases}x_{-} e\left(\pi_{-}, \pi, x_{-}\right)(\pi \cdot \zeta(z, \xi(1))) & \text { when } \pi_{-} \neq \pi \\ x_{-}(\pi \cdot \zeta(z, \xi(1))) & \text { when } \pi_{-}=\pi\end{cases}
$$

Obviously, $q$ is not weakly continuous as long as the constant term in transaction costs is non-null. Indeed, $X_{-}^{\pi_{-}}(1)-X_{-}^{\widetilde{\pi}}(1) \geq C$ for any $\tilde{\pi} \neq \pi_{-}$. Consider

$$
\eta\left(\pi_{-}, \pi, x_{-}, z\right)= \begin{cases}h(\pi, z), & \pi_{-}=\pi \\ h(\pi, z)+\ln e\left(\pi_{-}, \pi, x_{-}\right), & \pi_{-} \neq \pi\end{cases}
$$

The Bellman equation (18) can be written in an equivalent form

$$
v_{\beta}\left(\pi_{-}, x_{-}, z\right)=\sup _{\pi \in \mathcal{S}}\left\{\eta\left(\pi_{-}, \pi, x_{-}, z\right)+\beta \int v_{\beta} d q\left(\pi_{-}, x_{-}, z, \pi\right)\right\} .
$$

Let $a_{\beta}: \mathcal{H} \rightarrow \mathcal{S}$ be a measurable selector for $M v_{\beta}$ (see Lemma 4.1) and $I_{\beta}$ be the impulse region

$$
I_{\beta}=\left\{\left(\pi_{-}, x_{-}, z\right) \in \mathcal{H}: v_{\beta}\left(\pi_{-}, x_{-}, z\right)=M v_{\beta}\left(\pi_{-}, x_{-}, z\right)\right\}
$$


The optimal strategy in this formulation is given by a measurable function $f_{\beta}: \mathcal{H} \rightarrow \mathcal{S}$

$$
f_{\beta}\left(\pi_{-}, x_{-}, z\right)= \begin{cases}\pi_{-}, & \left(\pi_{-}, x_{-}, z\right) \notin I_{\beta}, \\ a_{\beta}\left(\pi_{-}, x_{-}, z\right), & \left(\pi_{-}, x_{-}, z\right) \in I_{\beta} .\end{cases}
$$

Since $v_{\beta}$ is unbounded as $\beta$ grows to $\infty$ we introduce the relative discounted value function

$$
w_{\beta}\left(\pi_{-}, x_{-}, z\right)=m_{\beta}-v_{\beta}\left(\pi_{-}, x_{-}, z\right),
$$

where

$$
m_{\beta}=\sup _{\pi_{-} \in \mathcal{S}} \sup _{z \in E} \widetilde{v}_{\beta}\left(\pi_{-}, z\right)
$$

is well-defined due to Lemma 4.6. Moreover, we have

LEMMA 5.3.

(i) $0 \leq w_{\beta}\left(\pi_{-}, x_{-}, z\right) \leq M_{1}+M_{2} \max \left(M_{3},-\ln x_{-}\right)$with $M_{1}, M_{2}, M_{3}$ $>0$ independent of $\beta, \pi_{-}, x_{-}, z$.

(ii) $\left\{(1-\beta) m_{\beta}: \beta \in(0,1)\right\}$ is a pre-compact set, i.e. its closure is compact.

Proof. By Lemma 4.6 and Corollary 4.8, we have $w_{\beta}\left(\pi_{-}, x_{-}, z\right) \leq m_{\beta}-v_{\beta}\left(\pi_{-}, z\right)+v_{\beta}\left(\pi_{-}, z\right)-v_{\beta}\left(\pi_{-}, x_{-}, z\right) \leq M+L\left(x_{-}\right)$, where $L\left(x_{-}\right)$is a function defined in Theorem 4.7. We conclude by using the form of $L\left(x_{-}\right)$. Part (ii) follows from boundedness of $h(\cdot)$ and $\ln \widetilde{e}(\cdot)$.

Put $\bar{\lambda}=\lim \sup _{\beta \uparrow 1}(1-\beta) m_{\beta}$, which is finite by Lemma 5.3(ii). Denote by $\beta_{k}$ a sequence of discount factors converging to 1 such that

$$
\bar{\lambda}=\lim _{k \rightarrow \infty}\left(1-\beta_{k}\right) m_{\beta_{k}} .
$$

Let

$$
\underline{w}(\vartheta)=\liminf _{k \rightarrow \infty, \vartheta^{\prime} \rightarrow \vartheta} w_{\beta_{k}}\left(\vartheta^{\prime}\right), \quad \vartheta \in \mathcal{H} .
$$

This can be written equivalently as

$$
\underline{w}(\vartheta)=\inf \left\{\liminf _{k \rightarrow \infty} w_{\beta_{k}}\left(\vartheta_{k}\right): \vartheta_{k} \rightarrow \vartheta\right\}, \quad \vartheta \in \mathcal{H} .
$$

Lemma 5.4 ([20, Lemma 3.1]). The function $\underline{w}$ is lower semicontinuous.

The proof of this lemma is straightforward and is based on the following reformulation of the definition of $\underline{w}$ :

$$
\underline{w}(\vartheta)=\sup _{n} \inf _{k \geq n}\left\{\inf _{\vartheta^{\prime} \in B(\vartheta, 1 / n)} w_{\beta_{k}}\left(\vartheta^{\prime}\right)\right\},
$$

where $B(\vartheta, 1 / n)$ is a ball in $\mathcal{H}$ of radius $1 / n$. 
In the following we use two transition operators corresponding to $q$. Let $\underline{q}$ be given by the formula (26) with

$$
X_{-}^{\pi}(1)=x_{-} e\left(\pi_{-}, \pi, x_{-}\right)(\pi \cdot \zeta(z, \xi(1)))
$$

and $\bar{q}$ with

$$
X_{-}^{\pi}(1)=x_{-}(\pi \cdot \zeta(z, \xi(1))) .
$$

They are weakly continuous. Indeed, it is straightforward by (A1) and the continuity of $e\left(\pi_{-}, \pi, x_{-}\right)$(see Lemma 2.1) that the mapping

$$
\left(\pi_{-}, x_{-}, z\right) \mapsto\left(\int_{\mathcal{H}} f d \underline{q}\left(\pi_{-}, x_{-}, z\right), \int_{\mathcal{H}} f d \bar{q}\left(\pi_{-}, x_{-}, z\right)\right)
$$

is continuous for any continuous bounded function $f: \mathcal{H} \rightarrow \mathbb{R}$.

Lemma 5.5 ([29, Lemma 3.2]). Let $\left\{\mu_{n}\right\}$ be a sequence of probability measures on a separable metric space $\mathcal{X}$ converging weakly to $\mu$ and $\left\{g_{n}\right\}$ be a sequence of measurable nonnegative functions on $\mathcal{X}$. Then

$$
\int \underline{g} d \mu \leq \liminf _{n \rightarrow \infty} \int g_{n} d \mu_{n}, \quad \text { where } \quad \underline{g}(x)=\liminf _{n \rightarrow \infty, y \rightarrow x} g_{n}(y), \quad x \in \mathcal{X} .
$$

THEOREM 5.6. Under assumptions (A1)-(A5) there exists a measurable function $f_{1}: \mathcal{H} \rightarrow \mathcal{S}$ and a measurable function $w: \mathcal{H} \rightarrow(-\infty, 0]$ such that

$$
w(\vartheta)+\bar{\lambda} \leq \eta\left(\vartheta, f_{1}(\vartheta)\right)+\int w\left(\vartheta^{\prime}\right) q\left(\vartheta, f_{1}(\vartheta)\right)\left(d \vartheta^{\prime}\right), \quad \vartheta \in \mathcal{H} .
$$

Proof. From equation (27) we derive

$$
\begin{array}{r}
w_{\beta}(\vartheta)+(\beta-1) m_{\beta}=-\eta\left(\vartheta, f_{\beta}(\vartheta)\right)+\beta \int w_{\beta}\left(\vartheta^{\prime}\right) q\left(\vartheta, f_{\beta}(\vartheta)\right)\left(d \vartheta^{\prime}\right), \\
\vartheta \in \mathcal{H}, \beta \in(0,1),
\end{array}
$$

where $f_{\beta}$ defines an optimal strategy for $v_{\beta}$. Fix $\vartheta \in \mathcal{H}$ and a sequence $\left(\vartheta_{k}\right)$ converging to $\vartheta$. The above equation can be rewritten as

$w_{\beta_{k}}\left(\vartheta_{k}\right)+\left(\beta_{k}-1\right) m_{\beta_{k}}=-\eta\left(\vartheta_{k}, f_{\beta_{k}}\left(\vartheta_{k}\right)\right)+\beta_{k} \int w_{\beta_{k}}\left(\vartheta^{\prime}\right) q\left(\vartheta_{k}, f_{\beta_{k}}\left(\vartheta_{k}\right)\right)\left(d \vartheta^{\prime}\right)$.

Applying $\liminf \operatorname{in}_{k \rightarrow \infty}$ to both sides yields

$$
\begin{aligned}
& \liminf _{k \rightarrow \infty} w_{\beta_{k}}\left(\vartheta_{k}\right)-\bar{\lambda} \\
& \quad=-\limsup _{k \rightarrow \infty} \eta\left(\vartheta, f_{\beta_{k}}\left(\vartheta_{k}\right)\right)+\liminf _{k \rightarrow \infty} \int \beta_{k} w_{\beta_{k}}\left(\vartheta^{\prime}\right) q\left(\vartheta_{k}, f_{\beta_{k}}\left(\vartheta_{k}\right)\right)\left(d \vartheta^{\prime}\right) .
\end{aligned}
$$

Since $\mathcal{S}$ is compact there exists a sequence $\left(n_{k}\right)$ such that $f_{\beta_{n_{k}}}(\vartheta) \rightarrow \pi^{*}$ and either (a) $\vartheta_{n_{k}} \in I_{\beta_{n_{k}}}$ for every $k$, or (b) $\vartheta_{n_{k}} \notin I_{\beta_{n_{k}}}$ for every $k$. Assume first that (a) holds. By virtue of Lemma 5.5 we have

$$
\liminf _{k \rightarrow \infty} \int \beta_{k} w_{\beta_{k}}\left(\vartheta^{\prime}\right) q\left(\vartheta_{k}, f_{\beta_{k}}\left(\vartheta_{k}\right)\right)\left(d \vartheta^{\prime}\right) \geq \int \underline{w}\left(\vartheta^{\prime}\right) \underline{q}\left(\vartheta, \pi^{*}\right)\left(d \vartheta^{\prime}\right) .
$$

By Corollary 4.5 the functions $v_{\beta}\left(\pi_{-}, x_{-}, z\right)$ are nondecreasing in $x_{-}$. This implies that $\underline{w}\left(\pi_{-}, x_{-}, z\right)$ is nonincreasing in $x_{-}$. Hence $\int \underline{w}\left(\vartheta^{\prime}\right) \underline{q}\left(\vartheta, \pi^{*}\right)\left(d \vartheta^{\prime}\right)$ 
$\geq \int \underline{w}\left(\vartheta^{\prime}\right) q\left(\vartheta, \pi^{*}\right)\left(d \vartheta^{\prime}\right)$ and

$$
\liminf _{k \rightarrow \infty} \int \beta_{k} w_{\beta_{k}}\left(\vartheta^{\prime}\right) q\left(\vartheta_{k}, f_{\beta_{k}}\left(\vartheta_{k}\right)\right)\left(d \vartheta^{\prime}\right) \geq \int \underline{w}\left(\vartheta^{\prime}\right) q\left(\vartheta, \pi^{*}\right)\left(d \vartheta^{\prime}\right) .
$$

In case (b) we have $f_{\beta_{n_{k}}}\left(\vartheta_{k}\right)=\pi_{-}^{k}$, where $\vartheta_{k}=\left(\pi_{-}^{k}, x_{-}^{k}, z^{k}\right)$. Obviously, $\pi^{*}=\pi_{-}$, where $\vartheta=\left(\pi_{-}, x_{-}, z\right)$. From the equalities $q\left(\vartheta, \pi^{*}\right)=\bar{q}\left(\vartheta, \pi^{*}\right)$ and $q\left(\vartheta_{n_{k}}, f_{\beta_{n_{k}}}\left(\vartheta_{k}\right)\right)=\bar{q}\left(\vartheta_{n_{k}}, f_{\beta_{n_{k}}}\left(\vartheta_{k}\right)\right)$ and Lemma 5.5 we obtain (30). Since $\eta$ is upper semicontinuous we conclude that

$$
\liminf _{k \rightarrow \infty} w_{\beta_{k}}\left(\vartheta_{k}\right)-\bar{\lambda} \geq-\eta\left(\vartheta, \pi^{*}\right)+\int \underline{w}\left(\vartheta^{\prime}\right) q\left(\vartheta, \pi^{*}\right)\left(d \vartheta^{\prime}\right) .
$$

Consequently,

$$
\liminf _{k \rightarrow \infty} w_{\beta_{k}}\left(\vartheta_{k}\right)-\bar{\lambda} \geq \inf _{\pi \in \mathcal{S}}\left\{-\eta(\vartheta, \pi)+\int \underline{w}\left(\vartheta^{\prime}\right) q(\vartheta, \pi)\left(d \vartheta^{\prime}\right)\right\} .
$$

Taking the infimum over all sequences $\vartheta_{n}$ converging to $\vartheta$ we finally obtain

$$
\underline{w}(\vartheta)-\bar{\lambda} \geq \inf _{\pi \in \mathcal{S}}\left\{-\eta(\vartheta, \pi)+\int \underline{w}\left(\vartheta^{\prime}\right) q(\vartheta, \pi)\left(d \vartheta^{\prime}\right)\right\} .
$$

To complete the proof we have to show that there exists a measurable selector for the infimum on the right-hand side of (31). Corollary 4.5 implies that $\underline{w}$ is non-increasing in $x_{-}$. Thus, for $\left(\pi_{-}, x_{-}, z\right) \in \mathcal{H}$,

$$
\int \underline{w}\left(\vartheta^{\prime}\right) \bar{q}\left(\pi_{-}, x_{-}, z, \pi_{-}\right)\left(d \vartheta^{\prime}\right) \leq \int \underline{w}\left(\vartheta^{\prime}\right) \underline{q}\left(\pi_{-}, x_{-}, z, \pi_{-}\right)\left(d \vartheta^{\prime}\right),
$$

and the infimum in (31) can be equivalently written as

$$
\begin{aligned}
\min \left\{-\eta\left(\vartheta, \pi_{-}\right)+\int \underline{w}\left(\vartheta^{\prime}\right)\right. & \bar{q}\left(\vartheta, \pi_{-}\right)\left(d \vartheta^{\prime}\right), \\
& \left.\inf _{\pi \in \mathcal{S}}\left\{-\eta(\vartheta, \pi)+\int \underline{w}\left(\vartheta^{\prime}\right) \underline{q}(\vartheta, \pi)\left(d \vartheta^{\prime}\right)\right\}\right\},
\end{aligned}
$$

where $\vartheta=\left(\pi_{-}, x_{-}, z\right)$. Recall that by Lemma 5.4 the function $\underline{w}$ is lower semicontinuous. By weak continuity of the transition probabilities $\underline{q}, \bar{q}$ the mappings

$$
\begin{aligned}
& \left(\pi_{-}, x_{-}, z, \pi\right) \mapsto \int_{\mathcal{H}} \underline{w}\left(\vartheta^{\prime}\right) \underline{q}\left(\pi_{-}, x_{-}, z, \pi\right)\left(d \vartheta^{\prime}\right), \\
& \left(\pi_{-}, x_{-}, z, \pi\right) \mapsto \int_{\mathcal{H}} \underline{w}\left(\vartheta^{\prime}\right) \bar{q}\left(\pi_{-}, x_{-}, z, \pi\right)\left(d \vartheta^{\prime}\right)
\end{aligned}
$$

are lower semicontinuous (see [14, Lemma 3.3(a)]). Corollary 1 in [7] implies that there exists a measurable selector $f_{2}: \mathcal{H} \rightarrow \mathcal{S}$ for

$$
\inf _{\pi \in \mathcal{S}}\left\{-\eta(\vartheta, \pi)+\int \underline{w}\left(\vartheta^{\prime}\right) \underline{q}(\vartheta, \pi)\left(d \vartheta^{\prime}\right)\right\} .
$$


Define $f_{1}: \mathcal{H} \rightarrow \mathcal{S}$ by

$f_{1}\left(\pi_{-}, x_{-}, z\right)$

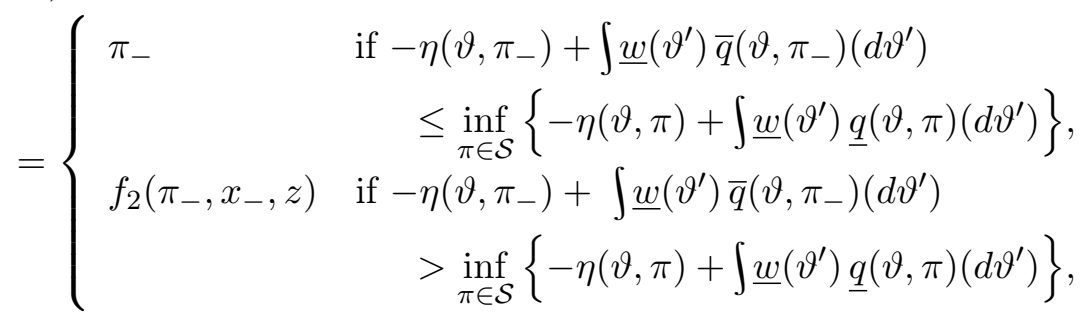

and put $w=-\underline{w}$. This completes the proof.

Fix $\left(\pi_{-}, x_{-}, z\right) \in \mathcal{H}$ and define a portfolio $\Pi=\left(\left(\pi_{1}, \tau_{1}\right),\left(\pi_{2}, \tau_{2}\right), \ldots\right)$ by the formulas of Theorem 5.1 with $I=\left\{\left(\pi_{-}, x_{-}, z\right) \in \mathcal{H}: f_{1}\left(\pi_{-}, x_{-}, z\right) \neq \pi_{-}\right\}$ and $p=f_{1}$. Iterating (28) $T$ times, dividing by $T$ and letting $T \rightarrow \infty$ we obtain

$$
\bar{\lambda} \leq J^{\pi_{-}, x_{-}, z}(\Pi)+\liminf _{T \rightarrow \infty} \mathbb{E}^{z} \frac{w\left(\pi_{-}^{\Pi}(T), X_{-}^{\Pi}(T), Z(T)\right)}{T} \leq J^{\pi_{-}, x_{-}, z}(\Pi),
$$

since $w$ is nonpositive. On the other hand, by a well-known Tauberian relation,

$$
\begin{aligned}
J^{\pi_{-}, x_{-}, z}(\Pi) & \leq \liminf _{\beta \rightarrow 1}(1-\beta) J_{\beta}^{\pi_{-}, x_{-}, z}(\Pi) \leq \liminf _{\beta \rightarrow 1}(1-\beta) v_{\beta}\left(\pi_{-}, x_{-}, z\right) \\
& \leq \liminf _{\beta \rightarrow 1}(1-\beta) v_{\beta}\left(\pi_{-}, z\right) \leq \bar{\lambda}
\end{aligned}
$$

which proves the optimality of $\Pi$ and completes the proof of Theorem 5.1.

Proof of Corollary 5.2. First notice that $\bar{\lambda}$ is the optimal value for the problem with proportional transaction costs. Indeed, if in the proof of Theorem 5.6 we put $w_{\beta}\left(\pi_{-}, z\right)=m_{\beta}-\widetilde{v}_{\beta}\left(\pi_{-}, z\right)$, we obtain an analog of $(28)$ with $w$ depending on $\pi_{-}, z$ and $\bar{\lambda}$ as above. Consequently, $\bar{\lambda}$ is the optimal value for the problem with proportional transaction costs, and the corresponding optimal strategy depends only on the current state of the processes $\left(\pi_{-}(t)\right)$ and $(Z(t))$.

Let $\Pi$ be the optimal portfolio for the case with fixed and proportional transaction costs (as defined in Theorem 5.1). Denote by $\widetilde{X}_{-}^{\Pi}(t)$ the wealth of the portfolio governed by $\Pi$ when the fixed term of the transaction cost function is equal to 0 . Obviously $\widetilde{X}_{-}^{\Pi}(t) \geq X_{-}^{\Pi}(t)$ and

$$
\lim _{T \rightarrow \infty} \frac{1}{T} \mathbb{E}^{z} \ln \widetilde{X}_{-}^{\Pi}(t) \geq \bar{\lambda}
$$

Since $\bar{\lambda}$ is the optimal value for the problem with proportional transaction costs we also have the opposite inequality.

(iii) follows directly from the proof of Theorem 4.7. 
6. Extensions. The paper can be extended in two directions. First we can generalize the cost function $\widetilde{c}$. Assume that $\widetilde{c}$ is subadditive and satisfies

$$
\begin{aligned}
& \widetilde{c}\left(N_{1}, N_{2}, S\right) \geq \sum_{i=1}^{d}\left(c_{i}^{1} S^{i}\left(N_{1}^{i}-N_{2}^{i}\right)^{+}+c_{i}^{2} S^{i}\left(N_{1}^{i}-N_{2}^{i}\right)^{-}\right), \\
& \widetilde{c}\left(N_{1}, N_{2}, S\right) \leq \sum_{i=1}^{d}\left(c_{i}^{1} S^{i}\left(N_{1}^{i}-N_{2}^{i}\right)^{+}+c_{i}^{2} S^{i}\left(N_{1}^{i}-N_{2}^{i}\right)^{-}\right)+C
\end{aligned}
$$

for some $C \geq 0$ and $c_{i}^{1}, c_{2}^{i} \in[0,1), i=1, \ldots, d$. If the cost function on the right-hand side of (34) satisfies (A6) then there exists an optimal portfolio of the form given in Theorem 5.1. Moreover, the portfolio optimal for the cost

$$
\sum_{i=1}^{d}\left(c_{i}^{1} S^{i}\left(N_{1}^{i}-N_{2}^{i}\right)^{+}+c_{i}^{2} S^{i}\left(N_{1}^{i}-N_{2}^{i}\right)^{-}\right)+C
$$

is optimal for $\widetilde{c}$ as well. To see this, denote by $\widehat{J}^{\pi_{-}, x_{-}, z}(\Pi)$ the functional (15) for the cost function $\widetilde{c}$, by $J^{\pi_{-}, x_{-}, z}(\Pi)$ the functional (15) for the cost function (35), and finally by $\widetilde{J}^{\pi_{-}, z}(\Pi)$ the functional (15) for the cost function (this a proportional cost)

$$
\sum_{i=1}^{d}\left(c_{i}^{1} S^{i}\left(N_{1}^{i}-N_{2}^{i}\right)^{+}+c_{i}^{2} S^{i}\left(N_{1}^{i}-N_{2}^{i}\right)^{-}\right) .
$$

Clearly, for any portfolio $\Pi \in \mathcal{A}^{z}$ we have

$$
\widetilde{J}^{\pi_{-}, z}(\Pi) \geq \widehat{J}^{\pi_{-}, x_{-}, z}(\Pi) \geq J^{\pi_{-}, x_{-}, z}(\Pi) .
$$

This implies that

$$
\sup _{\Pi \in \mathcal{A}^{z}} \widetilde{J}^{\pi_{-}, z}(\Pi) \geq \sup _{\Pi \in \mathcal{A}^{z}} \widehat{J}^{\pi_{-}, x_{-}, z}(\Pi) \geq \sup _{\Pi \in \mathcal{A}^{z}} J^{\pi_{-}, x_{-}, z}(\Pi) .
$$

Since by virtue of Theorem 5.1 and Corollary 5.2 there exists a constant $\lambda$ such that

$$
\lambda=\sup _{\Pi \in \mathcal{A}^{z}} \widetilde{J}^{\pi_{-}, z}(\Pi)=\sup _{\Pi \in \mathcal{A}^{z}} J^{\pi_{-}, x_{-}, z}(\Pi)
$$

we conclude that $\lambda=\sup _{\Pi \in \mathcal{A}^{z}} \widehat{J}^{\pi_{-}, x_{-}, z}(\Pi)$. Moreover, due to Corollary 5.2 the optimal portfolio for the functional $J^{\pi_{-}, x_{-}, z}$ is also optimal for $\widetilde{J}^{\pi_{-}, z}$. Therefore, it is also optimal for $\widehat{J}^{\pi_{-}, x_{-}, z}$. Notice now that the cost function (5) satisfies (33) and (34). Therefore, Theorem 5.1 extends to this important case.

The results of this paper can be applied to an incomplete information case and extend [25]. Let us first sketch some motivation for this development. It is well known that investors do not have full information about 
variables influencing the economy. This is due to errors in statistical data or simply due to the lack of information. Therefore, it is natural to extend our model to cover the case where a number of economic factors are either observable with noise or not observable at all. For simplicity we restrict ourselves to the case when a group of factors can be precisely observed and the rest are not observable. However, our results can be extended to a more general situation.

Following the above remark assume that the space $E$ of economic factors is a direct sum of metric spaces $E^{1}, E^{2}$ with Borel $\sigma$-algebras $\mathcal{E}^{1}, \mathcal{E}^{2}$. Therefore, $(Z(t))$ has a unique decomposition into $\left(Z^{1}(t), Z^{2}(t)\right)$. We shall treat $E^{1}$ as the observable part of the economic factor space and $\left(Z^{1}(t)\right)$ as the observable factor process. The process $\left(Z^{2}(t)\right)$ is the unobservable factor process. We denote by $\mathcal{M}_{t}, \mathcal{Z}_{t}^{1}, \mathcal{Z}_{t}^{2}$ the filtrations generated, respectively, by $(\zeta(t)),\left(Z^{1}(t)\right)$ and $\left(Z^{2}(t)\right)$. Denote by $\mathcal{Y}_{t}$ the filtration generated by $\mathcal{M}_{t}$ and $\mathcal{Z}_{t}^{1}$ and by $\tilde{\mathcal{A}}^{z}$ the space of $\mathcal{Y}_{t}$-adapted portfolios admissible for $z$, i.e. $\widetilde{\mathcal{A}}^{z} \subseteq \mathcal{A}^{z}$. Our aim is to prove existence of an optimal strategy maximizing the functional

$$
J^{\pi_{-}, x_{-}, z^{1}, \varrho}(\Pi)=\liminf _{T \rightarrow \infty} \frac{1}{T} \mathbb{E}^{z^{1}, \varrho} \ln X_{-}^{\Pi}(T)
$$

over all strategies $\Pi \in \widetilde{\mathcal{A}}$. Here $\left(z^{1}, \varrho\right) \in E^{1} \times \mathcal{P}\left(Z^{2}\right)$ denotes the initial distribution of $\left(Z^{1}(t), Z^{2}(t)\right)$ and $\mathcal{P}\left(Z^{2}\right)$ stands for the space of probability measures on $\left(Z^{2}, \mathcal{E}^{2}\right)$. Now, we can follow a similar reasoning as in [25] to apply Theorem 5.1 and prove existence of an optimal portfolio. Here, however, we improve several aspects of the result; firstly, the transaction costs structure covers important examples (4) and (5). The model setting is more general. Moreover, in [25] the space $E^{2}$ has to be compact to guarantee that $\mathcal{P}\left(E^{2}\right)$ is locally compact. Here, due to a different method of proof of Theorem 5.1 we allow $E^{2}$ to be a general complete separable metric space (in this case, $\mathcal{P}\left(E^{2}\right)$ is also a complete separable metric space). For further details see [25].

\section{Appendix}

Proof of Lemma 4.3. The first inequality is a direct consequence of the second. Set $\delta=e\left(\pi_{-}, \pi, x_{-}\right)$. By Lemma $2.1, \delta \geq 0$ and $c\left(\pi_{-}, \delta \pi\right)+C / x_{-}+$ $\delta \geq 1$. Noticing that $c\left(\pi_{-}, \delta \pi\right) \leq d \sum_{i=1}^{d}\left|\pi_{-}^{i}-\delta \pi^{i}\right|$ we obtain

$$
1 \leq d(1-\delta)+2 d \delta+\frac{C}{x_{-}}+\delta,
$$

which easily leads to the desired inequality.

Proof of Lemma 4.4. We shall prove (i) by contradiction: assume that $\widetilde{e}\left(\pi_{-}, \pi\right)<e\left(\pi_{-}, \pi, x_{-}\right)$. Noticing $a^{+}-b^{+} \leq(a-b)^{+}$and $a^{-}-b^{-} \leq(a-b)^{-}$ 
we obtain

$$
\left|c\left(\pi_{-}, \delta_{2} \pi\right)-c\left(\pi_{-}, \delta_{1} \pi\right)\right| \leq\left|\delta_{2}-\delta_{1}\right| \max _{i}\left(c_{i}^{1}, c_{i}^{2}\right) \quad \text { for } \delta_{1}, \delta_{2} \in[0,1] .
$$

Moreover, we have

$$
0 \leq e\left(\pi_{-}, \pi, x_{-}\right)-\widetilde{e}\left(\pi_{-}, \pi\right) \leq\left(e\left(\pi_{-}, \pi, x_{-}\right)-\widetilde{e}\left(\pi_{-}, \pi\right)\right) \max _{i}\left(c_{i}^{1}, c_{i}^{2}\right)-\frac{C}{x_{-}} .
$$

This gives the estimate

$$
1+\frac{C}{x_{-}\left(e\left(\pi_{-}, \pi, x_{-}\right)-\widetilde{e}\left(\pi_{-}, \pi\right)\right)} \leq \max _{i}\left(c_{i}^{1}, c_{i}^{2}\right)
$$

which contradicts the assumption that $c_{i}^{1}, c_{i}^{2} \in[0,1)$. The proof of $e\left(\pi_{-}, \pi, x_{-}\right)$ $\leq e\left(\pi_{-}, \pi, \widetilde{x}_{-}\right)$is analogous. Statement (ii) follows immediately from the inequality

$$
\widetilde{e}\left(\pi_{-}, \pi\right)-e\left(\pi_{-}, \pi, x_{-}\right) \leq\left(\widetilde{e}\left(\pi_{-}, \pi\right)-e\left(\pi_{-}, \pi, x_{-}\right)\right) \max _{i} c_{i}^{1}+\frac{C}{x_{-}} .
$$

For (iii) we apply the inequality $\ln (1+x) \leq x$ for $x>0$.

Proof of Corollary 4.5. For given $\pi_{-} \in \mathcal{S}, z \in E$ and $\widetilde{x}_{-} \leq x_{-}$

$$
v_{\beta}\left(\pi_{-}, \widetilde{x}_{-}, z\right)-v_{\beta}\left(\pi_{-}, x_{-}, z\right) \leq \sup _{\Pi \in \mathcal{A}^{z}}\left\{J_{\beta}^{\pi_{-}, \widetilde{x}_{-}, z}(\Pi)-J_{\beta}^{\pi_{-}, x_{-}, z}(\Pi)\right\} .
$$

Therefore, the result follows from the observation that $J_{\beta}^{\pi_{-}, \widetilde{x}_{-}, z}(\Pi)-$ $J_{\beta}^{\pi_{-}, x_{-}, z}(\Pi) \leq 0$ for any $\pi \in \mathcal{A}^{z}$.

Proof of Lemma 4.6. Let $\underline{e}=\inf _{\pi_{-}, \pi \in \mathcal{S}} \widetilde{e}\left(\pi_{-}, \pi\right)$. Since $\max _{i}\left(c_{i}^{1}, c_{i}^{2}\right)<1$, we have $\underline{e}>0$. Fix $z, z^{\prime} \in E$ and $\pi_{-}, \pi_{-}^{\prime} \in \mathcal{S}$. Denote by $\Pi$ the portfolio optimal for $\widetilde{v}_{\beta}\left(\pi_{-}, z\right)$, and by $\Pi^{\prime}$ the one optimal for $\widetilde{v}_{\beta}\left(\pi_{-}^{\prime}, z^{\prime}\right)$ (they exist by Theorem 4.2). The corresponding proportion processes $\pi_{-}^{\Pi, z}(t), \pi_{-}^{\Pi^{\prime}, z^{\prime}}(t)$ will be written as $\pi_{-}(t), \pi_{-}^{\prime}(t)$ and the corresponding wealth processes $X_{-}^{\Pi, z}(t)$, $X_{-}^{\Pi^{\prime}, z^{\prime}}(t)$ as $X_{-}(t), X_{-}^{\prime}(t)$. We then have

$$
\begin{aligned}
\widetilde{v}_{\beta}\left(\pi_{-}, z\right)- & \widetilde{v}_{\beta}\left(\pi_{-}^{\prime}, z^{\prime}\right) \\
= & \sum_{t=0}^{n-1} \beta^{t} \mathbb{E}^{z} h\left(\pi_{-}(t), z(t)\right)+\sum_{k=1}^{\infty} \mathbb{E}^{z}\left\{1_{\tau_{k}<n} \beta^{\tau_{k}} \ln \widetilde{e}\left(\pi_{-}\left(\tau_{k}\right), \pi_{k}\right)\right\} \\
& -\sum_{t=0}^{n-1} \beta^{t} \mathbb{E}^{z^{\prime}} h\left(\pi_{-}^{\prime}(t), z^{\prime}(t)\right)-\sum_{k=1}^{\infty} \mathbb{E}^{z^{\prime}}\left\{1_{\tau_{k}<n} \beta^{\tau_{k}} \ln \widetilde{e}\left(\pi_{-}^{\prime}\left(\tau_{k}\right), \pi_{k}\right)\right\} \\
& +\beta^{n}\left(\mathbb{E}^{z} \widetilde{v}_{\beta}\left(\pi_{-}(n), z(n)\right)-\mathbb{E}^{z^{\prime}} \widetilde{v}_{\beta}\left(\pi_{-}^{\prime}(n), z^{\prime}(n)\right)\right) .
\end{aligned}
$$

There are at most $n$ transactions between 0 and $n-1$, since by subadditivity of the cost function it is never optimal to have more than one transaction at 
a time. Hence,

$$
\begin{aligned}
& \widetilde{v}_{\beta}\left(\pi_{-}, z\right)-\widetilde{v}_{\beta}\left(\pi_{-}^{\prime}, z^{\prime}\right) \\
& \quad \leq n\|h\|_{\mathrm{sp}}-n \ln \underline{e}+\beta^{n}\left(\mathbb{E}^{z} \widetilde{v}_{\beta}\left(\pi_{-}(n), z(n)\right)-\mathbb{E}^{z^{\prime}} \widetilde{v}_{\beta}\left(\pi_{-}^{\prime}(n), z^{\prime}(n)\right)\right),
\end{aligned}
$$

where $\|h\|_{\text {sp }}=\sup h-\inf h$ is a span seminorm. Choose an arbitrary $\pi^{*} \in \mathcal{S}$ and observe that

$$
\begin{aligned}
& \mathbb{E}^{z} \widetilde{v}_{\beta}\left(\pi_{-}(n), z(n)\right)-\mathbb{E}^{z^{\prime}} \widetilde{v}_{\beta}\left(\pi_{-}^{\prime}(n), z^{\prime}(n)\right) \\
& \leq \mathbb{E}^{z}\left\{\widetilde{v}_{\beta}\left(\pi_{-}(n), z(n)\right)-\widetilde{v}_{\beta}\left(\pi^{*}, z(n)\right)\right\} \\
& +\mathbb{E}^{z^{\prime}}\left\{\widetilde{v}_{\beta}\left(\pi^{*}, z^{\prime}(n)\right)-\widetilde{v}_{\beta}\left(\pi_{-}^{\prime}(n), z^{\prime}(n)\right)\right\} \\
& +\mathbb{E}^{z} \widetilde{v}_{\beta}\left(\pi^{*}, z(n)\right)-\mathbb{E}^{z^{\prime}} \widetilde{v}_{\beta}\left(\pi^{*}, z^{\prime}(n)\right) .
\end{aligned}
$$

Since $\widetilde{v}_{\beta}\left(\pi_{-}, z\right)-\widetilde{v}_{\beta}\left(\pi_{-}^{\prime}, z\right) \leq-\ln \widetilde{e}\left(\pi, \pi^{\prime}\right)$, we have

$$
\begin{array}{r}
\mathbb{E}^{z}\left\{\widetilde{v}_{\beta}\left(\pi_{-}(n), z(n)\right)-\widetilde{v}_{\beta}\left(\pi^{*}, z(n)\right)\right\} \leq-\ln \underline{e}, \\
\mathbb{E}^{z^{\prime}}\left\{\widetilde{v}_{\beta}\left(\pi^{*}, z^{\prime}(n)\right)-\widetilde{v}_{\beta}\left(\pi_{-}^{\prime}(n), z^{\prime}(n)\right)\right\} \leq-\ln \underline{e} .
\end{array}
$$

Notice that

$$
\mathbb{E}^{z} \widetilde{v}_{\beta}\left(\pi^{*}, z(n)\right)-\mathbb{E}^{z^{\prime}} \widetilde{v}_{\beta}\left(\pi^{*}, z^{\prime}(n)\right)=\int_{E} \widetilde{v}_{\beta}\left(\pi^{*}, y\right) q(d y),
$$

with $q=P^{n}(z, \cdot)-P^{n}\left(z^{\prime}, \cdot\right)$. Let $\Gamma \in \mathcal{E}$ be the set from the Hahn-Jordan decomposition of the signed measure $q$, i.e. $q$ is nonnegative on $\Gamma$ and nonpositive on $\Gamma^{c}$. By (A3),

$$
\int_{E} \widetilde{v}_{\beta}\left(\pi^{*}, y\right) q(d y) \leq\left\|\widetilde{v}_{\beta}\left(\pi^{*}, \cdot\right)\right\|_{\mathrm{sp}} q(\Gamma) \leq \kappa\left\|\widetilde{v}_{\beta}\left(\pi^{*}, \cdot\right)\right\|_{\mathrm{sp}} \cdot
$$

Consequently,

$$
\widetilde{v}_{\beta}\left(\pi_{-}, z\right)-\widetilde{v}_{\beta}\left(\pi_{-}^{\prime}, z^{\prime}\right) \leq n\|h\|_{\mathrm{sp}}-(n+2) \ln \underline{e}+\kappa\left\|\widetilde{v}_{\beta}\left(\pi^{*}, \cdot\right)\right\|_{\mathrm{sp}} .
$$

Since $\pi_{-}, \pi_{-}^{\prime} \in \mathcal{S}$ and $z, z^{\prime} \in E$ are arbitrary we obtain

$$
\left\|\widetilde{v}_{\beta}\left(\pi^{*}, \cdot\right)\right\|_{\mathrm{sp}} \leq n\|h\|_{\mathrm{sp}}-(n+2) \ln \underline{e}+\kappa\left\|\widetilde{v}_{\beta}\left(\pi^{*}, \cdot\right)\right\|_{\mathrm{sp}},
$$

which yields the desired result.

\section{References}

[1] K. Aase and B. Øksendal, Admissible investment strategies in continuous trading, Stoch. Proc. Appl. 30 (1988), 291-301.

[2] M. Akian, A. Sulem and M. Taksar, Dynamic optimization of long term growth rate for a portoflio with transaction costs - the logarithmic utility case, Math. Finance 11 (2001), 153-188.

[3] P. H. Algoet and T. M. Cover, Asymptotic optimality and asymptotic equipartition properties of log-optimum investment, Ann. Probab. 16 (1988), 876-898. 
[4] A. Arapostathis et al., Discrete-time controlled Markov processes with average cost criterion: a survey, SIAM J. Control Optim. 31 (1993), 282-344.

[5] T. R. Bielecki and S. R. Pliska, Risk sensitive dynamic asset management, Appl. Math. Optim. 37 (1999), 337-360.

[6] T. R. Bielecki, S. R. Pliska and M. Sherris, Risk sensitive asset allocation, J. Econ. Dyn. Control 24 (2004), 1145-1177.

[7] L. D. Brown and R. Purves, Measurable selections of extrema, Ann. Statist. 1 (1973), 902-912.

[8] M. D. Donsker and S. R. S. Varadhan, Asymptotic evaluation of certain Markov process expectations for large time, III, Comm. Pure Appl. Math. 29 (1976), 389461.

[9] J. L. Doob, Stochastic Processes, Wiley, 1953.

[10] D. Duffie, Dynamic Asset Pricing Theory, Princeton Univ. Press, 2001.

[11] T. Duncan, B. Pasik-Duncan and E. Stettner, Adaptive control of discrete time Markov processes by large deviations method, Appl. Math. (Warsaw) 27 (2000), $265-285$.

[12] W. H. Fleming and S. J. Sheu, Risk-sesitive control and an optimal investment model, Math. Finance 10 (2000), 197-213.

[13] L. Gerencsér, M. Rásonyi and Zs. Vágó, Log-optimal currency portfolios and control Lyapunov exponents, in: 44th IEEE Conference on Decision and Control and European Control Conference ECC 2005, 1746-1769.

[14] J. I. González-Trejo, O. Hernández-Lerma and L. F. Hoyos-Reyes, Minimax control of discrete-time stochastic systems, SIAM J. Control Optim. 41 (2003), 1626-1659.

[15] O. Hernández-Lerma and J. B. Lasserre, Discrete-Time Markov Control Processes, Springer, 1996.

[16] - - - Further Topics on Discrete-Time Markov Control Processes, Springer, 1999.

[17] A. Inoue and Y. Nakano, Optimal long-term investment model with memory, Appl. Math. Optim. 55 (2007), 93-122.

[18] G. Iyengar, Universal investment in markets with transaction costs, Math. Finance 15 (2005), 359-371.

[19] A. Jaśkiewicz and A. S. Nowak, On the optimality equation for the average cost Markov control processes with Feller transition probabilities, J. Math. Anal. Appl. 316 (2006), 495-509.

[20] - - - Zero-sum ergodic stochastic games with Feller transition probabilities, SIAM J. Control Optim. 45 (2006), 773-789.

[21] J. L. Kelly, A new interpretation of information rate, Bell System Techn. J. 35 (1956), 917-926.

[22] K. Kuroda and H. Nagai, Risk-sensitive portfolio optimization on infinite time horizon, Stoch. Stoch. Rep. 73 (2002), 309-331.

[23] R. Liptser, Large deviations for occupation measures of Markov proceses: Discrete time, noncompact case, Theory Probab. Appl. 41 (1996), 35-54.

[24] D. G. Luenberger, Investment Science, Oxford Univ. Press, 1998.

[25] J. Palczewski and $\mathrm{L}$. Stettner, Maximization of the portfolio growth rate under fixed and proportional transaction costs, Comm. Information Systems 7 (2007), 31-58.

[26] - , - Impulsive control of portfolios, Appl. Math. Optim. 56 (2007), 67-103.

[27] E. Platen, A benchmark approach to finance, Math. Finance 16 (2006), 131-151.

[28] M. Schäl, Average optimality in dynamic programming with general state space, Math. Oper. Res. 18 (1993), 163-172.

[29] R. Serfozo, Convergence of Lebesgue integrals with varying measures, Sankhyā Ser. A 44 (1982), 380-402. 
[30] Ł. Stettner, On impulsive control with long run average cost criterion, Studia Math. 76 (1983), 279-298.

[31] —, Discrete time risk sensitive portfolio optimization with consumption and proportional transaction costs, Appl. Math. (Warsaw) 32 (2005), 395-404.

[32] E. O. Thorp, Portfolio choice and the Kelly criterion, in: Stochastic Optimization Models in Finance, W. T. Ziemba and R. G. Wickson (eds.), Academic Press, New York, 1975, 599-619.

Jan Palczewski

School of Mathematics

University of Leeds

Leeds LS2 9JT, UK

and

Faculty of Mathematics

University of Warsaw

Banacha 2

02-097 Warszawa, Poland

E-mail: J.Palczewski@mimuw.edu.pl
Łukasz Stettner

Institute of Mathematics

Polish Academy of Sciences

Śniadeckich 8

00-956 Warszawa, Poland E-mail: stettner@impan.gov.pl

Received on 19.12.200\%;

revised version on 11.1.2008 
